\title{
Distribution of Boleteceous Mushrooms in India, Some New Records from Sal Forest of Central India
}

\author{
R. K. Verma and Vimal Pandro* \\ Forest Protection Division, Tropical Forest Research Institute, Jabalpur - 482 021, \\ Madhya Pradesh, India \\ *Corresponding author
}

A B S T R A C T

\section{Keywords}

Agaricomycetes,

Basidiomycota,

Boletales, distribution,

ecto-mycorrhiza,

edible mushroom

Article Info

Accepted:

18 May 2018

Available Online:

10 June 2018
An account of Boleteceous mushrooms reported from different part of India is given. Total 84 species of Boletaceae were compiled from literature with records of habitat, distribution and references. Boletus spp. are the most common (37 species) followed by Strobilomyces (9 species), Tylopilus (7 species), Boletellus (6 species), Xerocomus (5 species), Suillus (3 species), Chalciporus, Retiboletus and Pulveroboletus (2 species each), Australoboletus, Borofutus, Hemileccinum, Hortiboletus, Leccinum. Octaviania, Phylloporus, Retiboletus, Rhodactina, Suillellus, Xerocomellus (one species each). Among Indian states, Himachal Pradesh and Sikkim represent the most boletes mushroom (16 species each) followed, Meghalaya (13), West Bengal (11), Madhya Pradesh and Uttarakhand (7 each), Jammu \& Kashmir (5) Chhattisgarh and Kerala (4) and rest of states showed 3 or less number of species. Six species of boletes namely: Boletellus ananas, B. chrysenteroides, B. dissiliens, B. pseudochrysenteroides, B. corneri and Boletus edulis were recorded for the first time from sal forest of central India (Chhattisgarh and Madhya Pradesh). These fungi are known to form ectomycorrizal association with sal trees.

\section{Introduction}

Basidiomycetes places in family Boletaceae are mushrooms which are primarily characterized by developing their spores in small pores, instead of gills, as are found in agarics. Among these mushrooms, Boletus edulis which is also known as the king mushroom is of high demand by mushroom hunters. Typical members of the family are generally known as boletes. These are a relatively safe group of mushrooms for human consumption, as none of these are known to be deadly poisonous to adults. These are little confused with deadly mushrooms, like various Amanita agarics, which are the most poisonous mushrooms in the world. Due to absence of gills boletes can be easily distinguished from gilled mushrooms. These are also easily recognized having colorful caps, pores and thick stems. Most species in Boletaceae produce large fleshy mushrooms with a central stipe. In most of species, flesh that is bruised or cut turned blue as a result of the oxidation of pulvinic acid derivatives (Nelson, 2010). Boletes were first described by the French botanist François Fulgis Chevallier in 1826 in a family, distinct from 
Agaricaceae. According to the Dictionary of the Fungi (Kirk et al., 2008), 35 genera are recognized in boletaceae, which collectively contain 787 species. In the comprehensive work of (Wu et al., 2014), seven major clades at subfamily level and 59 generic lineages were uncovered, including four new subfamilies (Austroboletoideae, Chalciporoideae, Leccinoideae, and Zangioideae) and 22 new potential genera have been described. Boletes are found worldwide, on every continent except Antarctica. These fungi are well-known reported from temperate regions of northern hemisphere; newer research has also shown significant diversity in tropical and southern hemisphere regions as well. A large number of boletes are delicious or at least edible. On the other hand poisonous or inedible species also exist, however, such as the unpalatable bitter species, for example Boletus calopus and Tylopilus felleus (bitter bolete). Some orangecapped species of Leccinum are also inedible. Several guidebooks recommend avoiding all red-pored boletes, but both $B$. erythropus (Neoboletus luridiformis) and Suillellus luridus are edible when well-cooked. Some of the boletes genera were separated based on basidiospores morphology, for example Boletellus have olive brown elongate to fusoid with longitudinally grooves and winged basidiospores (almond like) while Boletus have smooth spores (Pegler and Young, 1981).

The present article reports distribution of 84 boletaceous mushroom in different states of India. Six boletes (Boletellus ananas, $B$. chrysenteroides, B. dissiliens, $B$. pseudochrysenteroides, B. corneri and Boletus edulis) were also reported for the first time from sal forest of central India.

\section{Materials and Methods}

Specimens of bolete mushrooms were collected from sal forest of Madhya Pradesh and Chhattisgarh states in rainy seasons from forest floor under sal trees. Some parts of collected samples were preserved in $70 \%$ alcohol just after collection for microscopic study. The fruit bodies of fungi were dried under the sun or in the wooden box lighted with $100 \mathrm{~W}$ electric bulb. Microscopic slides were prepared by using stain, mountant, clearing and softening chemicals. Micro slides were observed under advanced research microscope (Leica, Germany) using 5x, 10x, 20x, 40x objectives and 10x and 15x eyepieces. Observations under phase contrast and dark field were also made whenever required. Photomicrography was done with the help of a digital camera (make, Leica) attached to the advanced microscope. Identification of fungi have been done with the help of published literature, monographs, books, keys, etc. (Ahmad 1950; Berkeley $1851 \mathrm{a} ; \mathrm{b} ; 1852 \mathrm{a} ; \mathrm{b} ; 1854 \mathrm{a}$; Bhavanidevi and Nair 1983; Chaouhan et al., 2010; Cunningham, 1942; Dar et al., 2010; De, 2006; Harsh and Bisht, 1983, 1985; Kumar and Sharma, 2011; Lakhanpal and Sagar, 1989; Lakhanpal, 1996; Murrill, 1909; Pyasi et al., 2011; Shajahan and Samajpati, 1995; Sharma et al., 1978; Sharma and Lakhanpal, 1988; Singer and Singh, 1971; Singer, 1948; Singer and Singh, 1971; Tiwari et al., 2013; Verma et al., 2008; Wu et al., 2014, 2015; Zang et al., 2001).

\section{Results and Discussion}

\section{Taxonomic description}

\section{Boletellus ananas (M.A. Curtis) Murrill (Figure 1-6)}

$\equiv$ Boletus ananas M.A. Curtis

Pileus, convexo-plane, dry, dull crimson to rose red, often fading to pale fawn drab, finely tomentose, cracking in to large floccose squamules, 60-75mm in diameter and $22 \mathrm{~mm}$ 
thick. Margin far exceeding the pores and covering them as a false veil, then splitting radially and stellately, appendiculate. Stem, central, $120 \times 12 \mathrm{~mm}$, subcylindric, base often enlarged and villous with thick white mycelium, tomentose, tan buff or buff-white, base and apex of pinkish shade. Flesh, 10mm thick at the centre of the pileus, $4-5 \mathrm{~mm}$ halfway to the margin, creamish white with patches con-colorous with the pileus patches, soft to touch.

Hymenium, yellow, pores $1 / \mathrm{mm}$, angular, pore tube $12 \mathrm{~mm}$ long, yellow. Basidiospores, vinaceous chocolate brown, boletoid, longitudinally striate in the hyaline exospores, slightly curved, oblique main striae disappearing at the ends of the spore apiculate, 17.0-21.0 x 7.5-9.0 $\mu \mathrm{m}$. Basidia, long clavate,

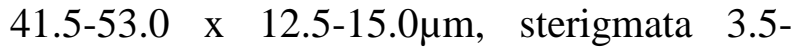
$5.5 \mu \mathrm{m}$. Cystidia, ventricose with obtuse wide apex, some with a subcylindric appendage, thin walled, hyaline. Tramal hyphae, hyaline thick-walled septate, 5.0-7.5 $\mu \mathrm{m}$ wide.

\section{Collections examined}

In humus of Shorea robusta, Nagadand forest, Sarguja, Chhattisgarh, 22/9/2011, Tropical Forest Research Institute TF 3196.

Boletellus chrysenteroides (Snell) Snell (Figures 7-10)

\section{$=$ Boletus chrysenteroides Snell}

Cap $4-11 \mathrm{~cm}$, convex to broadly convex with age, dry, finely velvety to nearly bald; sometimes becoming cracked with age; dark brown to nearly black at first, becoming medium brown or eventually pale brown. Stem, $2-10 \mathrm{~cm}$ long; up to $1.5 \mathrm{~cm}$ thick; more or less equal, at first punctuated by brownish, Leccinum-like scabers that later become aggregated into hairy or sub-scaly clusters that sometimes approximate the appearance of reticulation; yellowish to brownish at first, becoming reddish to purplish red in the midportion with age. Pore surface: bright to dull yellow, becoming olive yellow; bruising slowly blue and eventually brown; pore 1-2/ $\mathrm{mm}$ round to angular, tubes up to about $1 \mathrm{~cm}$ deep. Flesh pale yellow to whitish, or with age reddish in the mid-portion of the stem and around damaged areas; changing to bluish or blue when sliced.

$\mathrm{KOH}$ reaction, black on cap, brownish on flesh, iron salts olive on flesh. Spore print, olive-brown. Basidiospores 10-17 x 5-8 $\mu \mathrm{m}$; longitudinally twisted-grooved; ellipsoid; yellow in $\mathrm{KOH}$. Pileipellis a trichoderm; terminal elements often cystidioid, with subterminal elements sometimes somewhat inflated.

\section{Collection examined}

On soil surface in sal forest of Chada, Dindori, Madhya Pradesh, 25/07/2017, Tropical Forest Research Institute, TF 4041.

\section{Boletellus corneri Klofac \& Krisai (Figures 11-15)}

=Boletellus fallax (Corner) Watling

Pileus convex, pale rose red to pinkish brown, bearing rough angular cracks on its dorsal surface exposing white creamy flesh, bearing yellow angular pore tubes 3-5 mm long. Stalk long $9-15 \times 1-1.5 \mathrm{~cm}$, hard, characteristically bent, red rose to faint pink may be pinkish yellow with white mycelial tufts base. Spores

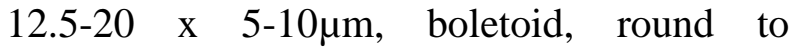
elongate, oblong with small apicules, longitudinally striated with slender ridges 7-10 in side view. Basidia 27.5-40 x 8.75-12.5 $\mu \mathrm{m}$, sterigmata 4, $(2.5-3.5 \mu \mathrm{m}$ long). Cystidia 45$160 \times 12.5-15.5 \mu \mathrm{m}$ ventrucose with prolong base $3.5-6.0 \mu \mathrm{m}$ wide and a projecting neck with obtuse to sub capitate apex 3-9 $\mu$ m wide. 


\section{Collection examined}

On forest floor of sal, Amarkantak, Madhya Pradesh, 23/08/2011, Mycology Herbarium, Tropical Forest Research Institute, Jabalpur TF 2649.

\section{Boletellus dissiliens (Corner) Pegler \& T.W.K. Young (Figures 16-21)}

$\equiv$ Boletus dissiliens Corner

Pileus: $45 \mathrm{~mm}$ pale pinkish buff cap, yellow cyanescent flesh, yellow, dull pinkish tan, subtomentose, dry, cracking into large flag patches, margin at first greatly extending the pores, covering them as a veil, splitting radically, stellately. Stem: $70 \mathrm{~mm}$ long, solid and $8 \mathrm{~mm}$ wide near the apex, $12 \mathrm{~mm}$ at base, at the thickened base villose with the white mycelium, hard, concolourous with the pileus, apex pallid. Pore tube: $5 \mathrm{~mm}$ long, sinuato adnate, ventricose, golden yellow then brownish ochraceous, cyanescent: pore angular, concolours, cyanescent. Flesh: $6 \mathrm{~mm}$ thick in the centre of the pileus. 3-4 mm halfway to the margin, white, pale yellowish

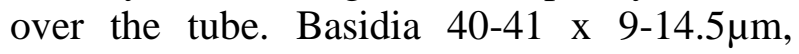
pyriform, sterigmata, $5.0-5.25 \mu \mathrm{m}$. Cystidia: $41.5 \times 17.68 \mu \mathrm{m}$ Basidiospores: olive brown in mass, ellipsoid boletoid, almond shape, rather coarsely striate with ridges, $13.5-17.5$ x 5.5 $8.5 \mu \mathrm{m}$.

\section{Collection examined}

On ground near base of Shorea robusta tree, Nagadand forest, Sarguja, Chhattisgarh, 22/9/2011, Tropical Forest Research Institute TF 3198.

Boletellus pseudochrysenteroides A.H. Sm. \& Thiers (Figures 22-28)

Growing alone, scattered, or gregariously. Cap convex, becoming broadly convex, 5-6cm; dry; soft; felty to velvety, becoming cracked, dark brick red, fading to pinkish brick red. Pore surface, yellowish at first, becoming olive, pores angular, 2-3 $\mathrm{mm}$ wide; pore-tubes up to $0.6-1.2 \mathrm{~cm}$ deep. Stem: $14-16 \mathrm{~cm}$ long; $1.6-1.8 \mathrm{~cm}$ thick; more or less equal; dry; solid; finely hairy; colored like the cap or paler, yellow at the apex; basal mycelium dense and whitish to yellowish. Flesh pale to bright yellow in the cap. Basidia, clavate, hyaline, 4sterigmate, rounded in sterigmatal part, measuring $17.5-26 \times 14-16.5 \mu \mathrm{m}$, sterigmata 2.5-3.7 $\mu \mathrm{m}$. Spore-print, brown to dark olive

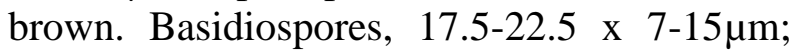
longitudinally striate, with 5-9 ridges, ellipsoid or nearly so, golden brown in $\mathrm{KOH}$. Diagnostic characters: The distinctive cap is brick red and soon prominently cracked up. The spores are ribbed or lined, which is characteristic of genus Boletellus. Similar species include reddish forms of Xerocomellus chrysenteron and Xerocomellus rubellus, Boletus chrysenteron, Boletellus chrysenteroides and Boletellus intermedius.

\section{Collection examined}

Growing under Shorea robusta tree, Amarkantak, Madhya Pradesh, 25/07/2017, Tropical Forest Research Institute TF 3986.

Boletus edulis Bull. (Figures 29-30)

$=$ Leccinum edule (Bulliard) Gray =Dictyopus edulis (Bulliard) Forquignon

Sporocarp small to medium sized. Pilus 5$3 \mathrm{~cm}$. diam., convex when young, broadly convex with age; surface dry, viscid when wet, glabrous, smooth, uneven colour brown, margin regular, smooth, incurved when young. Tubes 4-9 mm deep, adnexed but depressed around the stipe, violaceous grey when young. Pores minute, round, stuffed when young, pinkish brown to pale brown in age, unchanging on bruising. 
Fig.1-6 Boletellus ananas (1-2) Habit, fruitbody near sal tree (3) cystidia (4) hyphae (5) basidia with developing basidiospores on sterigmata (6) basidiospores
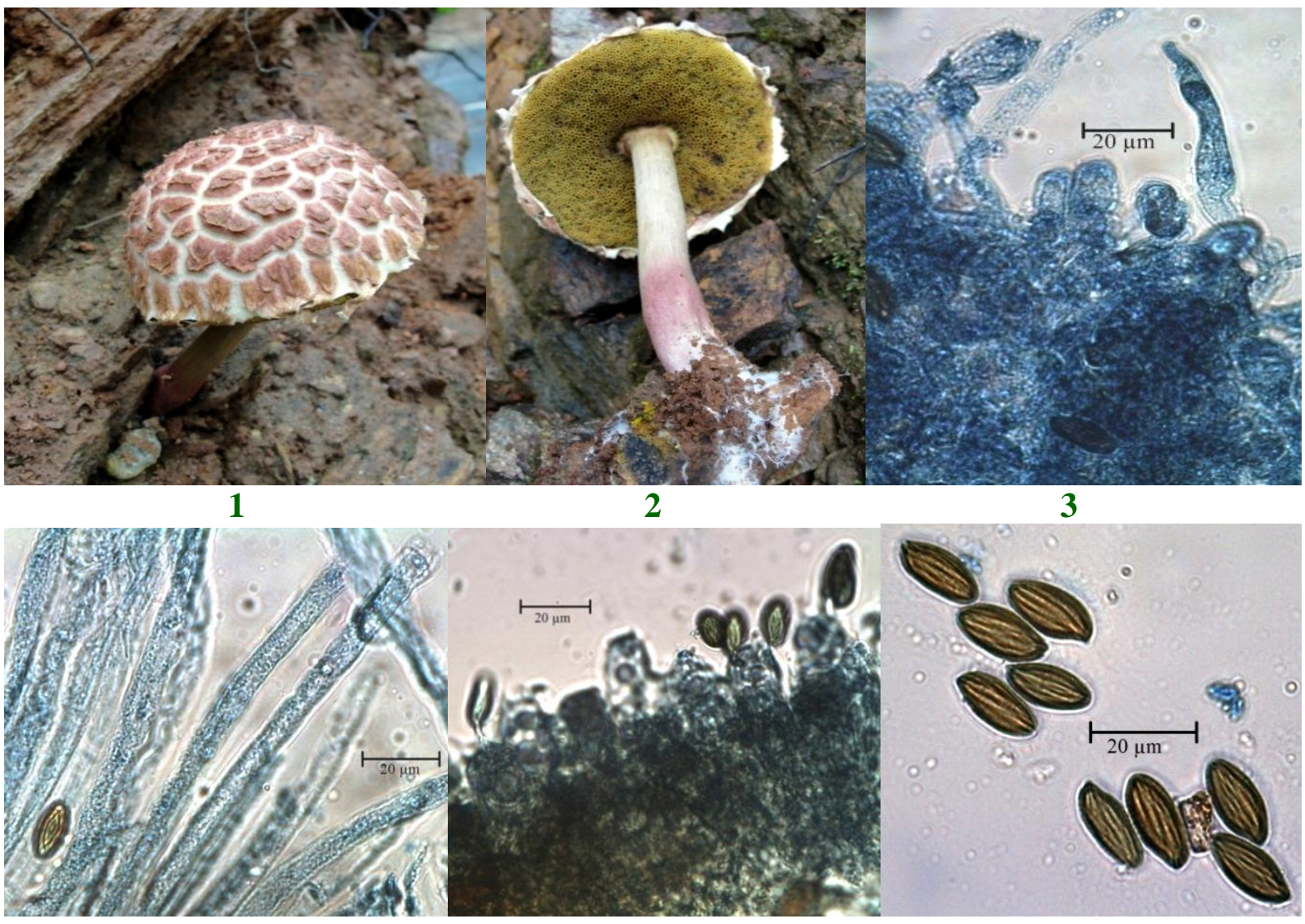

4

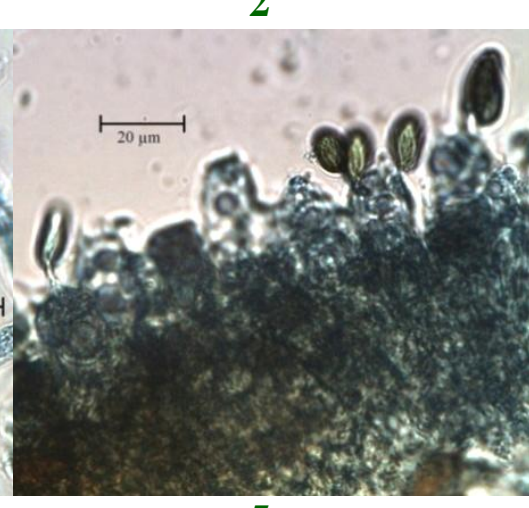

3

5

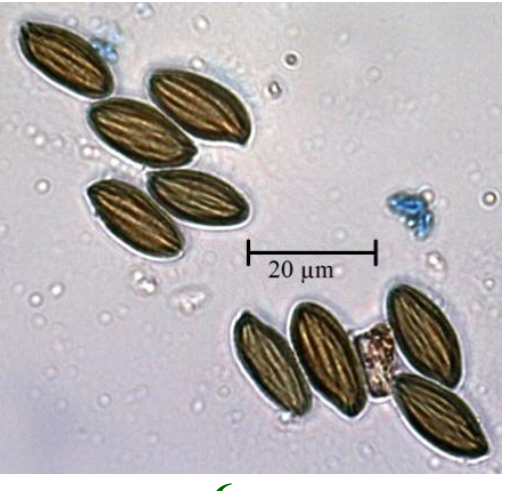

6

Fig.7-10 Boletellus chrysenteroides (7) habit, (8) sporophore showing pore surface, stipe and volva (9) basidiospores and hyphae and (10) basidiospores

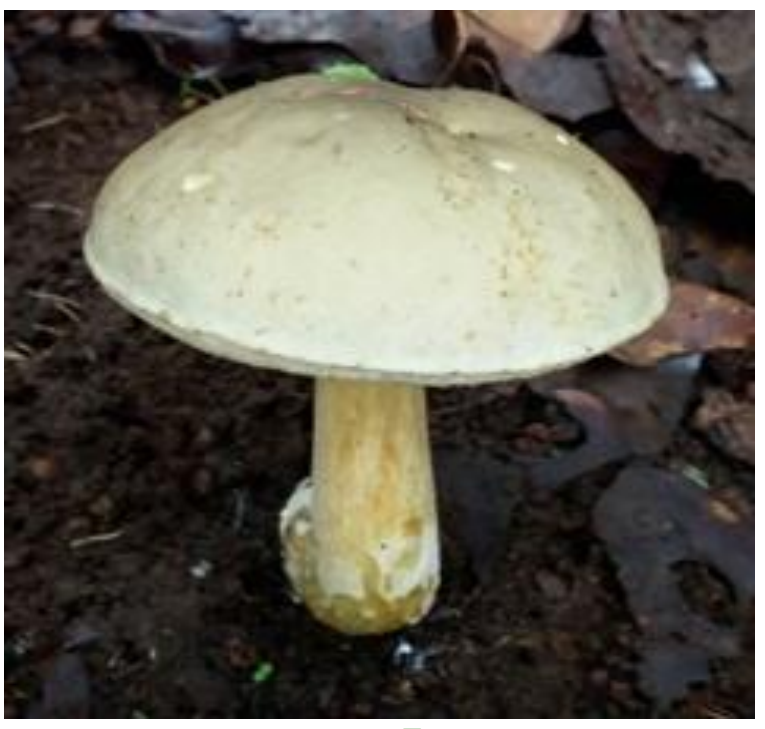

7

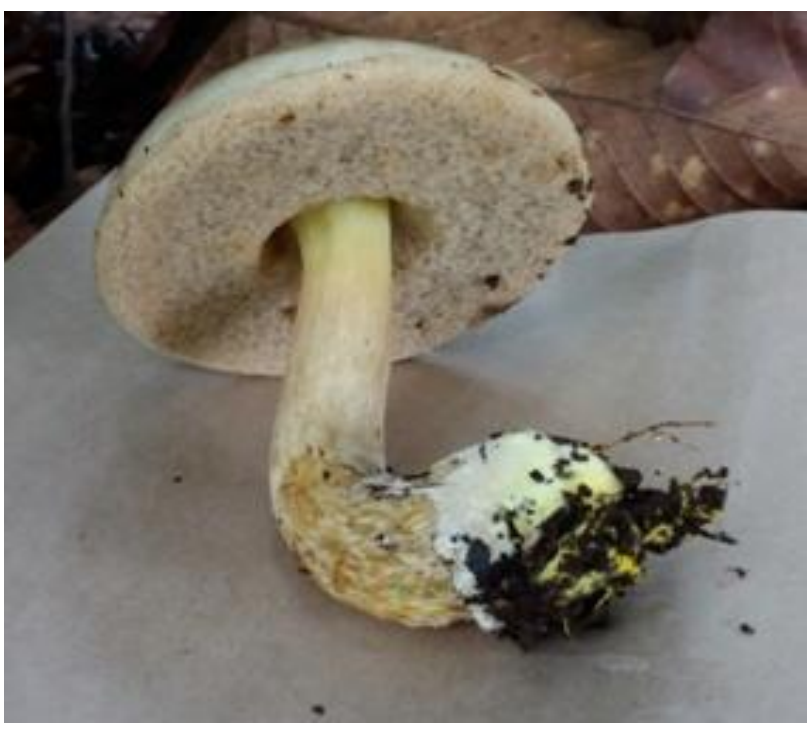

8 


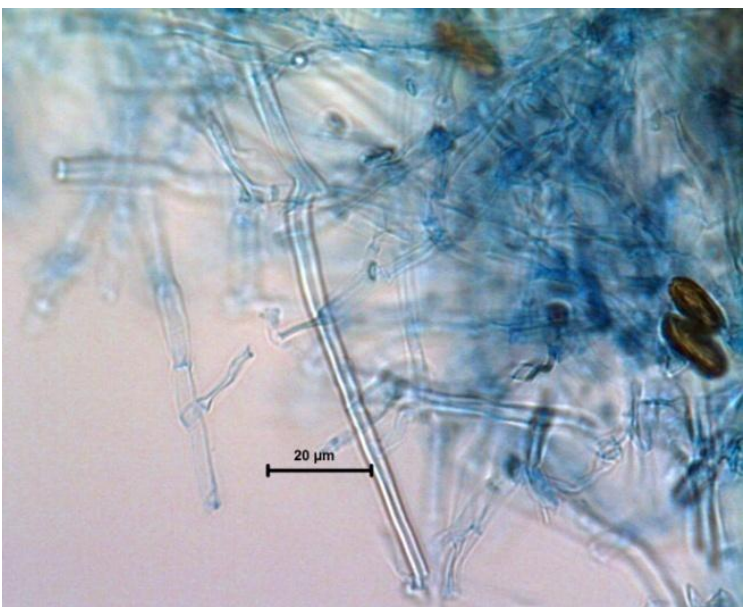

9

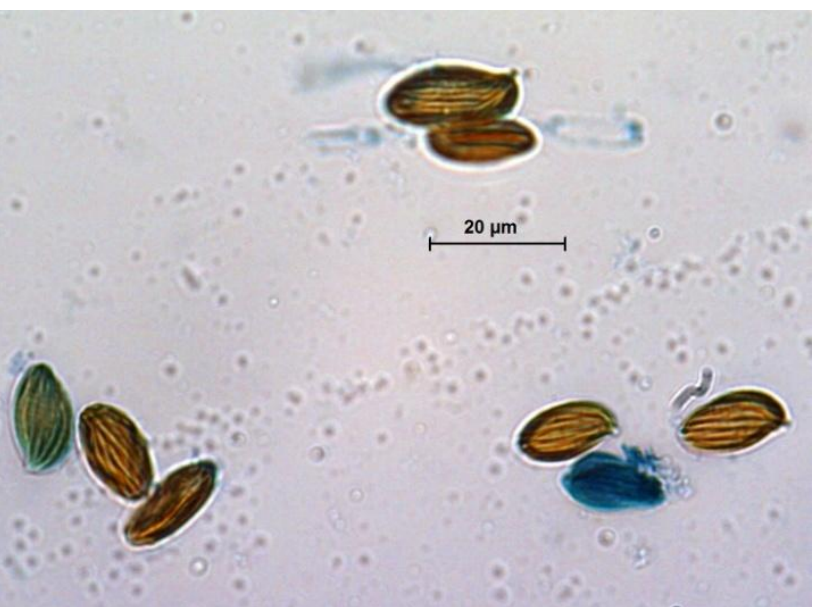

10

Fig.11-15 Boletellus corneri (11) fruitbody under sal tree (12) fruitbody pore surface, stioe and vulva (13) basidia with developing basidiospores (14) cystidia (15) basidiospores

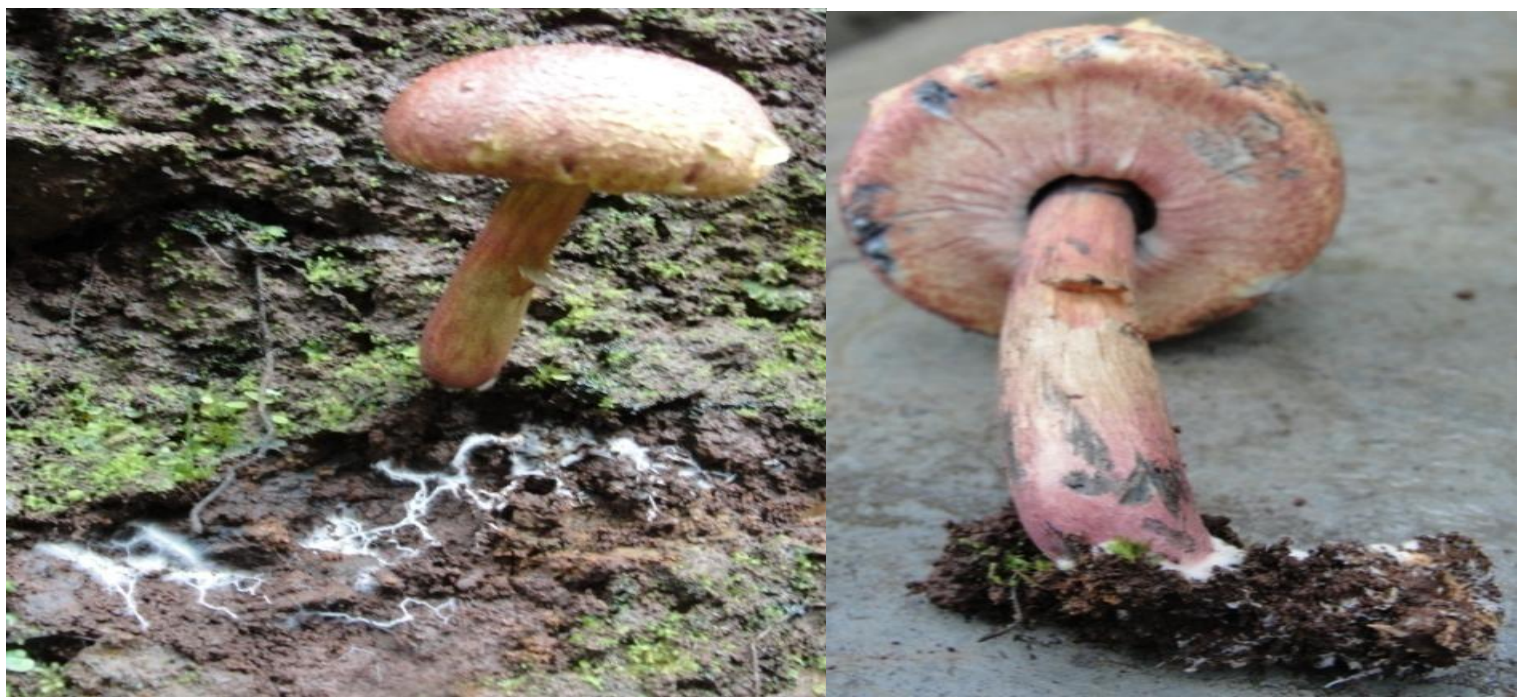

11

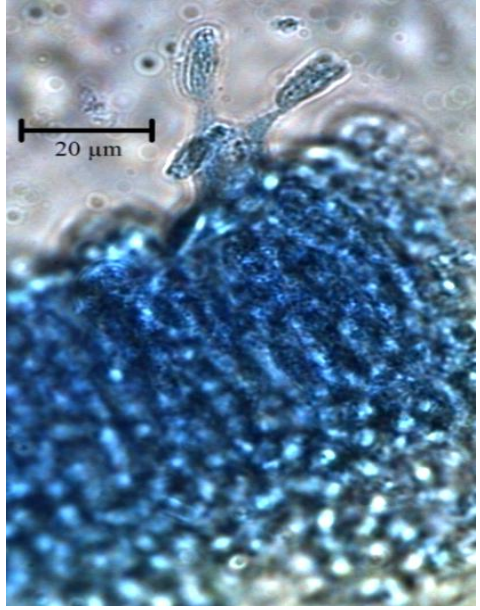

13

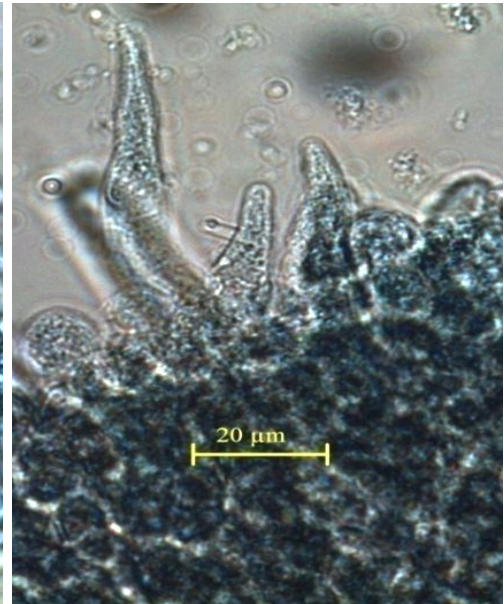

14
12

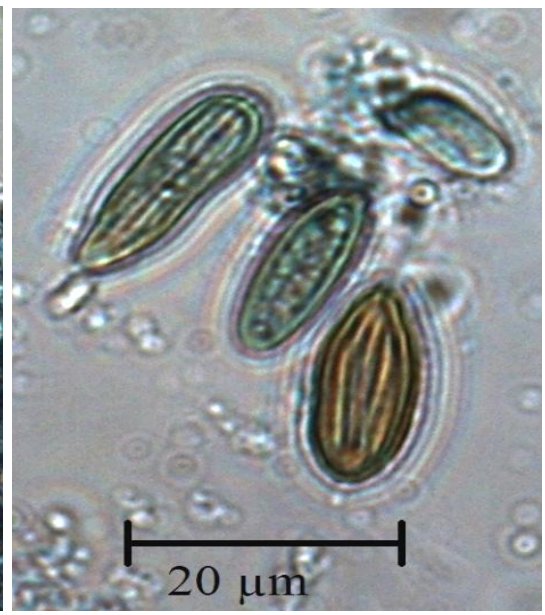

15 
Fig.16-21 Boletellus dissiliens (16) habit (17-18) sporphores under sal tree (19) hyphae (20) basidia with developing basidiospores (21) basidiospores
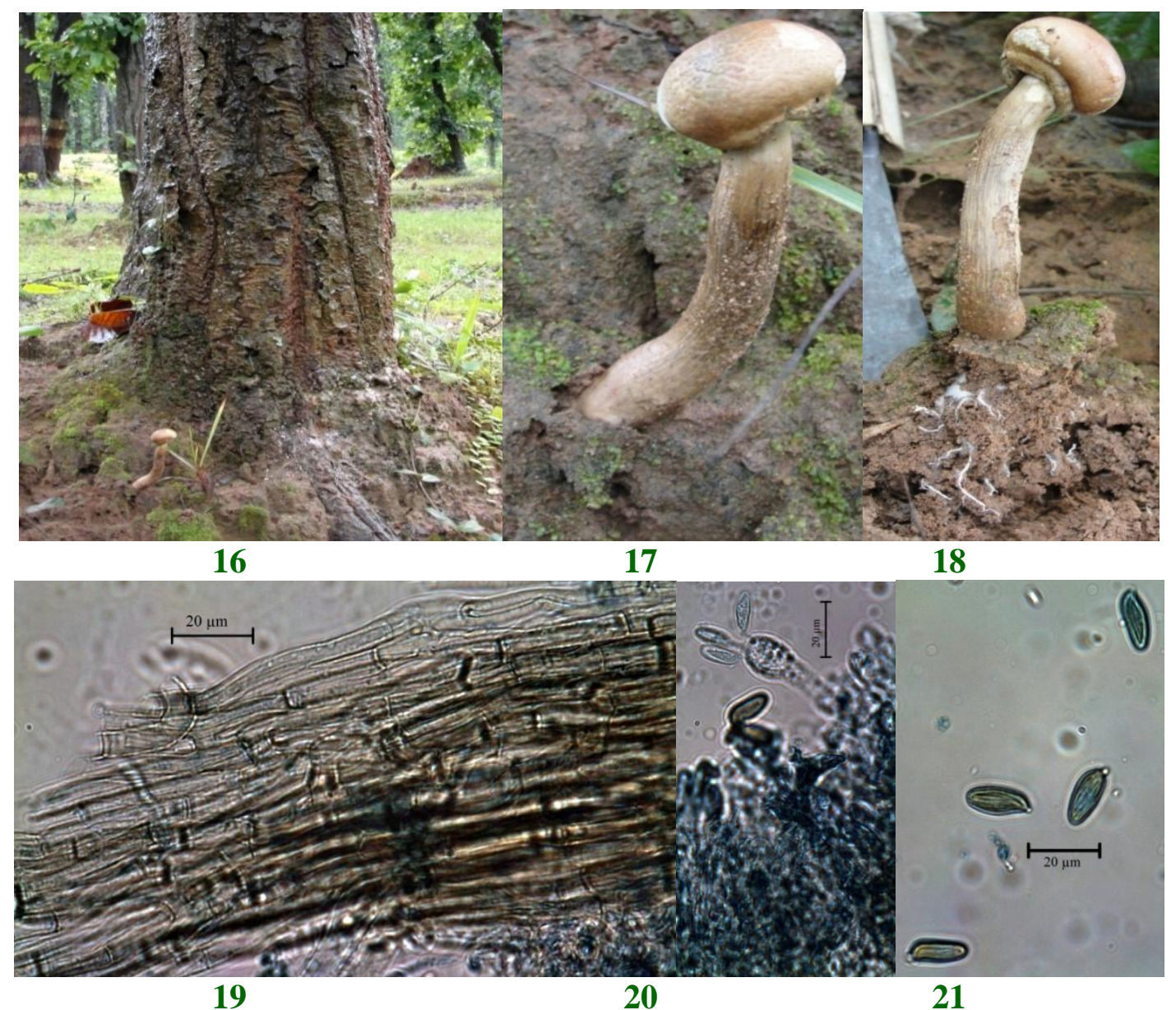

Fig.22-28 Boletellus pseudochrysenteroides. (22-24) habit (showing pileus, stem and pore surface covered with veil) (25) basidium (26) basidium attached with developing basidiospores and cystedia (27-28) basidiospores

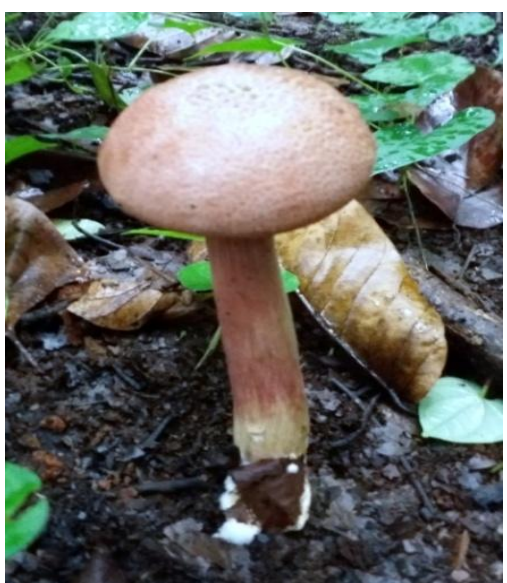

22

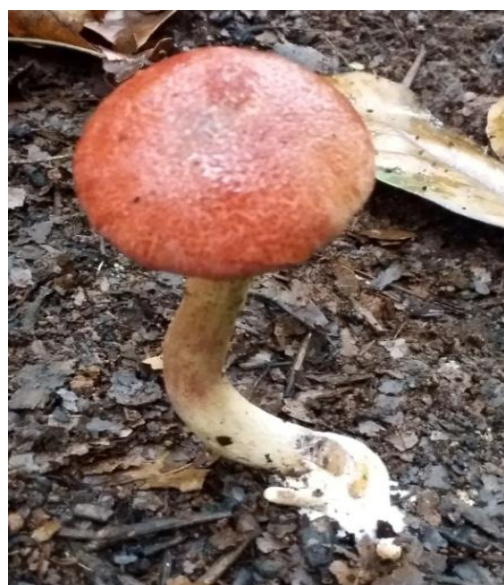

23

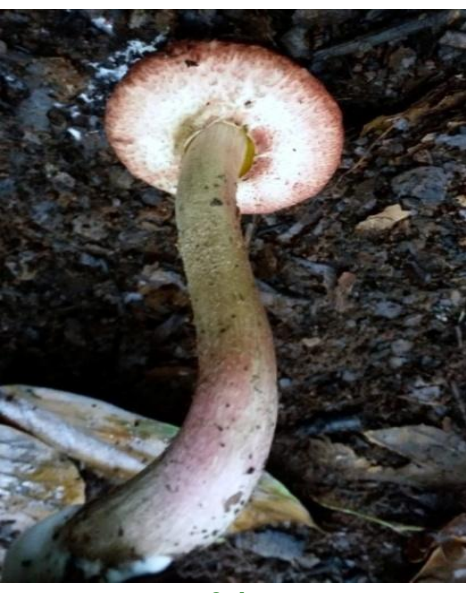

24 


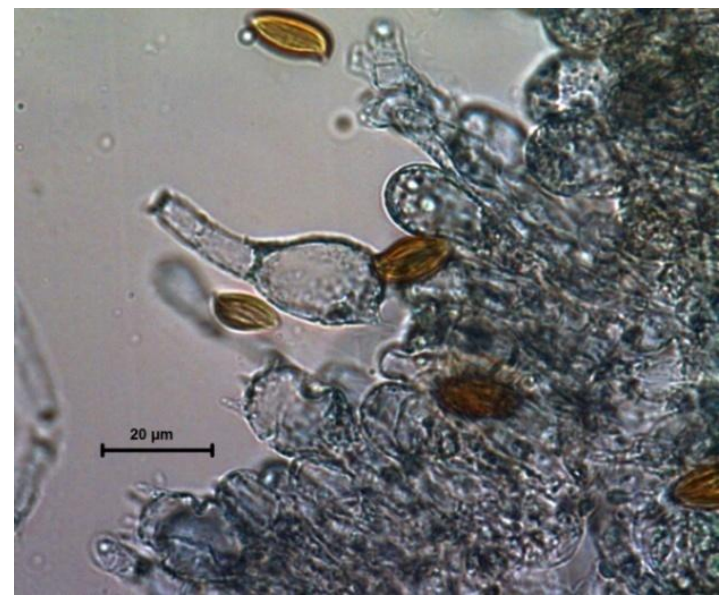

25

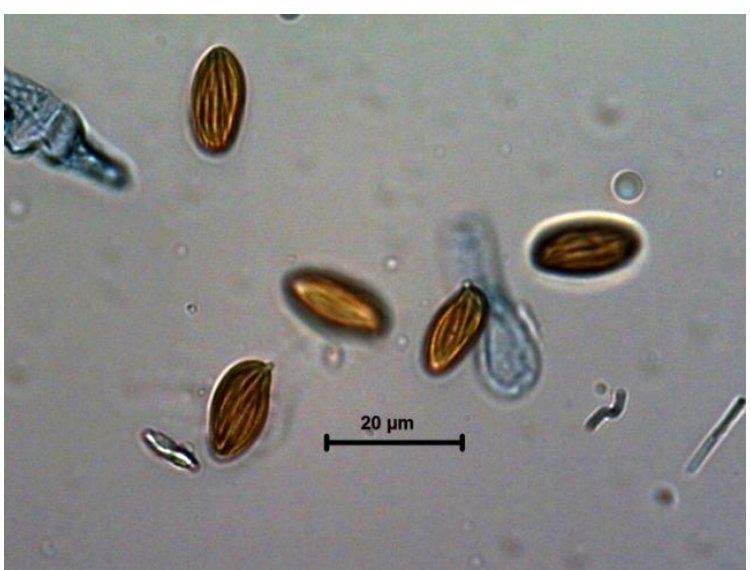

27

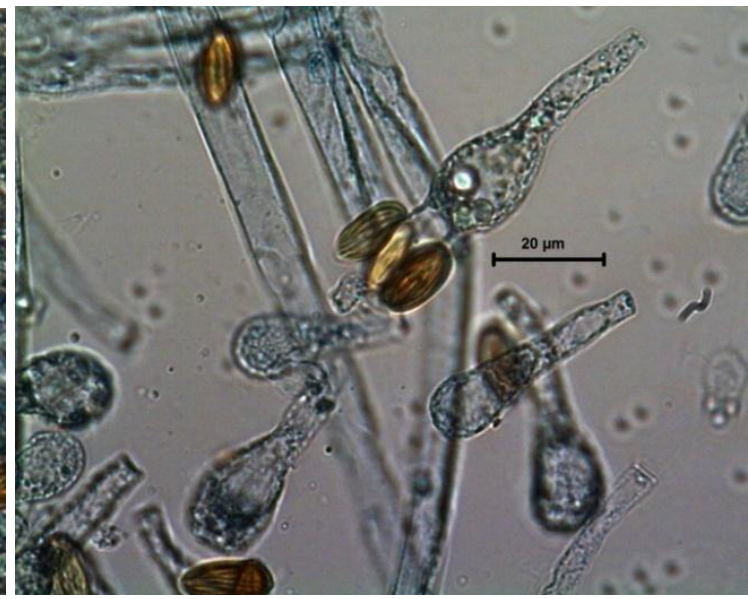

26

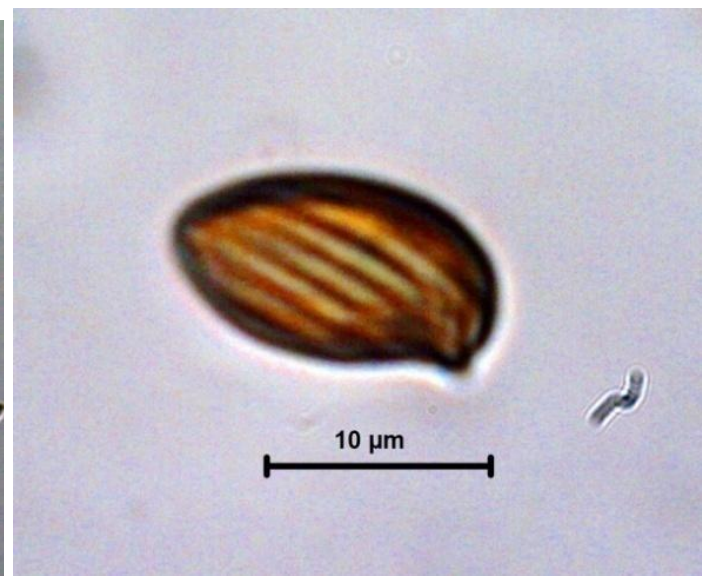

28

Fig.29-30 Boletus edulis (28) sporophore and (29) basidiospores
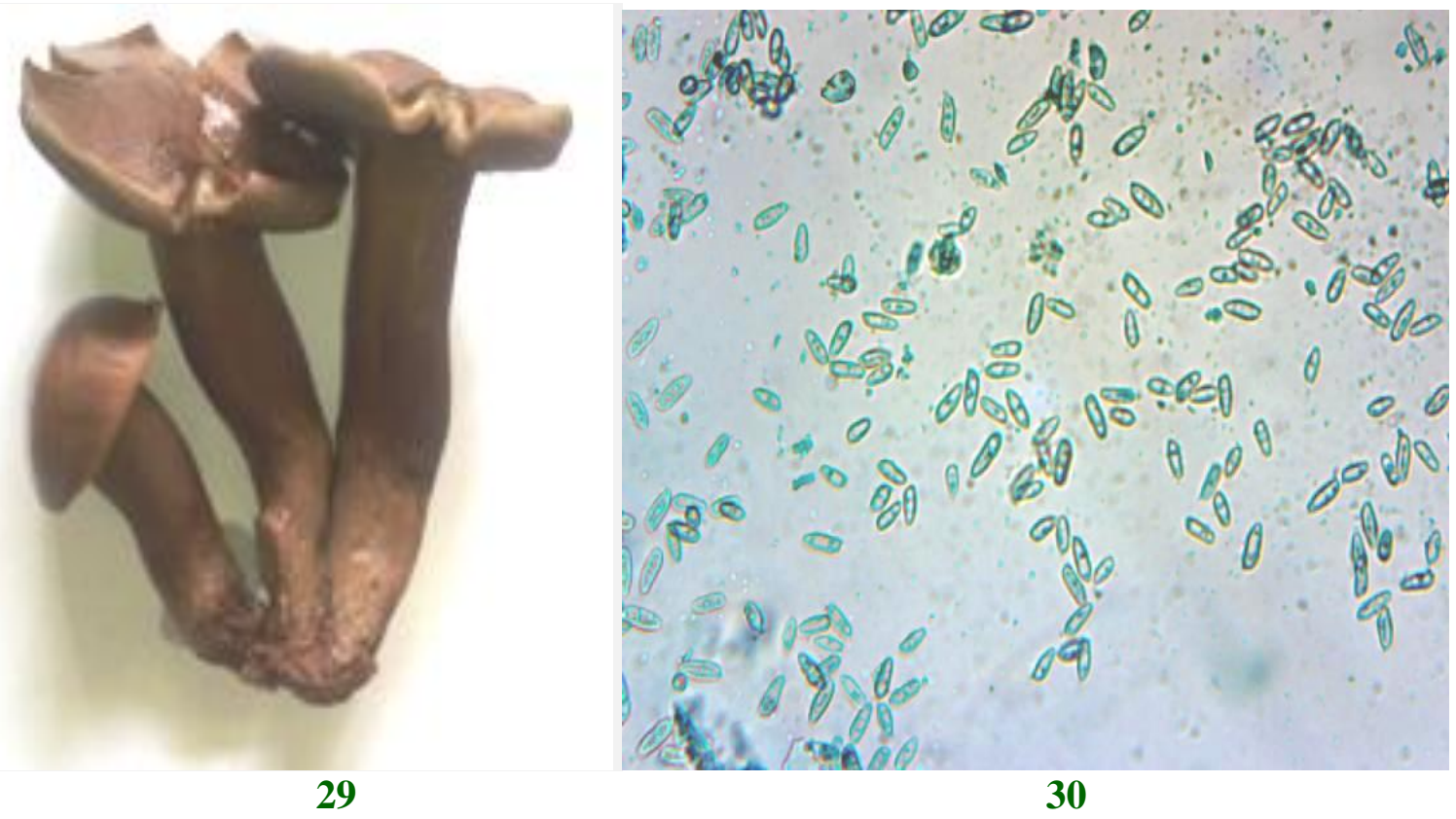
Fig.31 Distribution of Boletaceous mushroom fungi in India

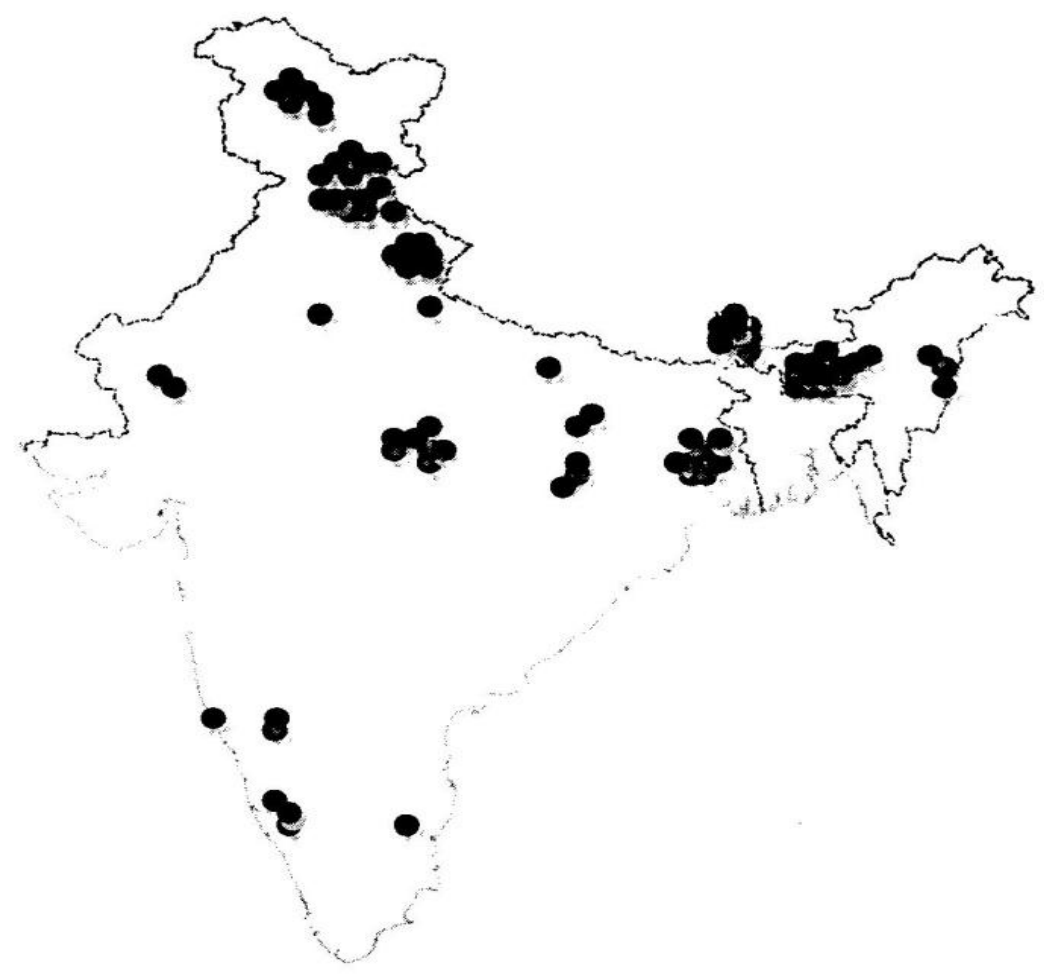

Table.1 Distribution of Boletaceous mushrooms in India

\begin{tabular}{|c|c|c|c|c|}
\hline S.N. & Species & Habitat & Distribution & Reference \\
\hline 1. & $\begin{array}{l}\text { Austroboletus } \\
\text { olivaceoglutinosus K. Das \& } \\
\text { Dentinger }\end{array}$ & $\begin{array}{l}\text { On soil associated with } \\
\text { Tsuga dumosa }\end{array}$ & Sikkim & $\begin{array}{l}\text { Das and Dentinger } \\
(2015)\end{array}$ \\
\hline 2. & $\begin{array}{l}\text { Boletellus ananas (M.A. } \\
\text { Curtis) Murrill }\end{array}$ & $\begin{array}{l}\text { In humus of sal forest } \\
\text { and on base of } \\
\text { Holigarna arnottiana }\end{array}$ & $\begin{array}{l}\text { Nagadand, Sarguja, } \\
\text { Chhattisgarh and } \\
\text { Thiruvananthapuram, } \\
\text { Kerala }\end{array}$ & $\begin{array}{l}\text { This article } \\
\text { Vrinda, Pradeep (2014) }\end{array}$ \\
\hline 3. & $\begin{array}{l}\text { Boletellus chrysenteroides } \\
\text { (Snell) Snell }\end{array}$ & $\begin{array}{l}\text { On soil surface in sal } \\
\text { forest }\end{array}$ & $\begin{array}{l}\text { Chada, Dindori, } \\
\text { Madhya Pradesh }\end{array}$ & This article \\
\hline 4. & $\begin{array}{l}\text { Boletellus corneri Klofac \& } \\
\text { Krisai }\end{array}$ & On forest floor of sal & $\begin{array}{l}\text { Amarkantak, Madhya } \\
\text { Pradesh }\end{array}$ & This article \\
\hline 5. & $\begin{array}{l}\text { Boletellus dissiliens (Corner) } \\
\text { Pegler \& T.W.K. Young }\end{array}$ & $\begin{array}{l}\text { On ground near base of } \\
\text { sal tree }\end{array}$ & $\begin{array}{l}\text { Nagadand, Sarguja, } \\
\text { Chhattisgarh }\end{array}$ & This article \\
\hline 6. & $\begin{array}{l}\text { Boletellus emodensis (Berk.) } \\
\text { Singer } \equiv \text { Boletus emodensis } \\
\text { Berk. }\end{array}$ & On forest soil & $\begin{array}{l}\text { Khasi Hills, } \\
\text { Meghalaya; } \\
\text { Darjeeling. West } \\
\text { Bengal and Sikkim }\end{array}$ & $\begin{array}{l}\text { Berkeley (1851a,b; 1852; } \\
\text { 1854) }\end{array}$ \\
\hline 7. & $\begin{array}{l}\text { Boletellus } \\
\text { pseudochrysenteroides A.H. } \\
\text { Sm. \& Thiers }\end{array}$ & $\begin{array}{l}\text { Growing under Shorea } \\
\text { robusta tree }\end{array}$ & $\begin{array}{l}\text { Amarkantak, Madhya } \\
\text { Pradesh }\end{array}$ & This article \\
\hline 8. & Boletus aestivalis Fr. & On ground in forest & Himachal Pradesh & Lakhanpal and Sagar \\
\hline
\end{tabular}




\begin{tabular}{|c|c|c|c|c|}
\hline & & & & (1989) \\
\hline 9. & $\begin{array}{l}\text { Boletus alexandri Sagar \& } \\
\text { T.N. Lakh. }\end{array}$ & On ground in forest & Himachal Pradesh & Lakhanpal (1996) \\
\hline 10. & $\begin{array}{l}\text { Boletus alutaceus Morgan } \\
=\text { Boletus alutaceus var. } \\
\text { simlensis T.N. Lakh. \& Sagar }\end{array}$ & $\begin{array}{l}\text { On brunt soil, and on } \\
\text { forest ground; } \\
\text { ectomycorrhizal with } \\
\text { Ficus benghalensis }\end{array}$ & $\begin{array}{l}\text { Simla, Himachal } \\
\text { Pradesh; } \\
\text { Goa }\end{array}$ & $\begin{array}{l}\text { Lakhanpal (1996) } \\
\text { Kamat et al., (2009) }\end{array}$ \\
\hline 11. & Boletus areolatus Berk. & Open pastures. & $\begin{array}{l}\text { Kala-Panee, Khasi } \\
\text { Hills, Meghalaya }\end{array}$ & Berkeley (1852a) \\
\hline 12. & Boletus chrysenteron Fries & $\begin{array}{l}\text { Ecto-mycorrhizal with } \\
\text { Holigarna arnotiana }\end{array}$ & $\begin{array}{l}\text { Thiruvananthapuram, } \\
\text { Kerala }\end{array}$ & Vrinda, Pradeep (2014) \\
\hline 13. & $\begin{array}{l}\text { Boletus cinerascens Schwein., } \\
=\text { Boletus cyanescens Bull. } \\
=\text { Gyroporus cyanescens } \\
\text { (Bull.) Quél. }\end{array}$ & On open places of earth & $\begin{array}{l}\text { Darjeeling, West } \\
\text { Bengal }\end{array}$ & Berkeley (1851b) \\
\hline 14. & Boletus craspedius Massee & On soil under oak forest & Kumaon, Uttarakhand & Harsh and Bisht, (1982b) \\
\hline 15. & $\begin{array}{l}\text { Boletus delphinus Hook.f. } \\
=\text { Boletus delphinus Berk. }\end{array}$ & On soil & $\begin{array}{l}\text { Darjeeling, West } \\
\text { Bengal }\end{array}$ & Berkeley (1851a) \\
\hline 16. & Boletus dissiliens Corner & On soil under oak forest & Kumaon, Uttarakhand & Harsh and Bisht, (1982b) \\
\hline 17. & Boletus edulis Bull. & $\begin{array}{l}\text { Mycorrhizal on sal, } \\
\text { growing on humicolous } \\
\text { soil in coniferous forest } \\
\text { and under Syzygium } \\
\text { cuminii; } \\
\text { coniferous forest; on } \\
\text { dead wood logs }\end{array}$ & $\begin{array}{l}\text { Amarkantak- } \\
\text { Achanakmar, Madhya } \\
\text { Pradesh and } \\
\text { Chhattisgarh and } \\
\text { Chishoti, Kishtwar, } \\
\text { J\&K; } \\
\text { forest of Khunti, } \\
\text { Jharkhand ; } \\
\text { Nagaland; Assam }\end{array}$ & $\begin{array}{l}\text { This article } \\
\text { Kumar and Sharma } \\
(2011 b) \\
\text { Srivastava et al., (2012) } \\
\text { Ao et al., (2016) } \\
\text { Sarma et al., (2010) }\end{array}$ \\
\hline 18. & Boletus emodensis Berk. & On the ground & $\begin{array}{l}\text { Darjeeling, West } \\
\text { Bengal }\end{array}$ & Berkeley (1851a) \\
\hline 19. & Boletus fallax Corner & $\begin{array}{l}\text { On forest floor of sal } \\
\text { forest }\end{array}$ & $\begin{array}{l}\text { Amarkantak- } \\
\text { Achanakmar, Madhya } \\
\text { Pradesh \& } \\
\text { Chhattisgarh }\end{array}$ & Pyasi et al., (2012) \\
\hline 20. & Boletus formosus Corner & $\begin{array}{l}\text { Growing in coniferous } \\
\text { mixed and broad leaved } \\
\text { forest }\end{array}$ & $\begin{array}{l}\text { Dugga, Bhadarwah, } \\
\text { Jammu \& Kashmir }\end{array}$ & $\begin{array}{l}\text { Kumar and Sharma } \\
\text { (2011b) }\end{array}$ \\
\hline 21. & Boletus frostii J.L. Russell & $\begin{array}{l}\text { On soil in pasture and } \\
\text { deodar tree }\end{array}$ & $\begin{array}{l}\text { Chail, Simla, } \\
\text { Himachal Pradesh }\end{array}$ & Sharma et al., (1978) \\
\hline 22. & Boletus furfuraceus Berk. & On clay-banks & $\begin{array}{l}\text { Moflong, Khasi Hills, } \\
\text { Meghalaya }\end{array}$ & Berkeley (1852a) \\
\hline 23. & Boletus gigas Berk. & $\begin{array}{l}\text { On soil and copses of } \\
\text { Andromeda and Birch }\end{array}$ & $\begin{array}{l}\text { Khasi Hills, } \\
\text { Meghalaya and } \\
\text { Lachen river, Sikkim }\end{array}$ & $\begin{array}{l}\text { Berkeley (1852a); Horak } \\
\text { (1980) }\end{array}$ \\
\hline 24. & Boletus gracilis Peck. & $\begin{array}{l}\text { On soil rich in humus } \\
\text { mixed forest }\end{array}$ & $\begin{array}{l}\text { Chambaghat, Solan, } \\
\text { Himachal Pradesh }\end{array}$ & Sharma et al., (1978) \\
\hline 25. & Boletus granulatus L. & $\begin{array}{l}\text { Growing on } \\
\text { humicolous soil in } \\
\text { scattered coniferous } \\
\text { forest }\end{array}$ & $\begin{array}{l}\text { Dugga, Bhadarwah, } \\
\text { Jammu \& Kashmir }\end{array}$ & $\begin{array}{l}\text { Kumar and Sharma } \\
\text { (2011b) }\end{array}$ \\
\hline 26. & Boletus griseus Frost & On soil & $\begin{array}{l}\text { Kandaghat, Solan, } \\
\text { Himachal Pradesh }\end{array}$ & Sharma et al., (1978) \\
\hline 27. & $\begin{array}{l}\text { Boletus hongoi T.N. Lakh. \& } \\
\text { Sagar }\end{array}$ & On ground in forest & Himachal Pradesh & Lakhanpal (1996) \\
\hline
\end{tabular}




\begin{tabular}{|c|c|c|c|c|}
\hline 28. & Boletus illudens Peck & On grassland & West Bengal & De (2006b) \\
\hline 29. & $\begin{array}{l}\text { Boletus lakhanpalii K. Das, D. } \\
\text { Chakr., Baghela, Sanjay K. } \\
\text { Singh \& Dentinger }\end{array}$ & $\begin{array}{l}\text { On soil, associated with } \\
\text { Larix griffithiana }\end{array}$ & Sikkim & Das et al., (2015) \\
\hline 30. & Boletus luridus Schaeff & $\begin{array}{l}\text { Growing on } \\
\text { humicolous soil in } \\
\text { scattered mixed forest }\end{array}$ & $\begin{array}{l}\text { Bhadarwah, Jammu \& } \\
\text { Kashmir }\end{array}$ & $\begin{array}{l}\text { Kumar and Sharma } \\
\text { (2011b) }\end{array}$ \\
\hline 31. & Boletus niveus Jullien ex Vill. & On soil, mixed forest & $\begin{array}{l}\text { Kandaghat, Solan, } \\
\text { Himachal Pradesh }\end{array}$ & Sharma et al., (1978) \\
\hline 32. & Boletus parvulus (Paulet) Lév. & $\begin{array}{l}\text { On soil rich in humus } \\
\text { mixed forest }\end{array}$ & $\begin{array}{l}\text { Chambaghat, Solan, } \\
\text { Himachal Pradesh }\end{array}$ & Sharma et al., (1978) \\
\hline 33. & $\begin{array}{l}\text { Boletus pusillus Berk. } \equiv \text { Suillus } \\
\text { pusillus Kuntze }\end{array}$ & On ground & $\begin{array}{l}\text { Moflong, Khasi Hills, } \\
\text { Meghalaya }\end{array}$ & Berkeley (1854a) \\
\hline 34. & $\begin{array}{l}\text { Boletus rhodoxanthes } \\
\text { (Krombh) Kallenb. }\end{array}$ & $\begin{array}{l}\text { From moist humus soil } \\
\text { under conifer } \\
\text { dominated forest }\end{array}$ & Gulmarg, Kashmir & Dar et al., (2010) \\
\hline 35. & Boletus rubripes Thiers & $\begin{array}{l}\text { Growing under } \\
\text { Picea spinulosa } \\
\text { Forest }\end{array}$ & $\begin{array}{l}\text { North district, } \\
\text { Dombang valley, } \\
\text { Sikkim, }\end{array}$ & Das (2013a) \\
\hline 36. & Boletus scaber Bull. & On soil & Himachal Pradesh & Sharma et al., (1978) \\
\hline 37. & Boletus scrobiculatus Berk. & $\begin{array}{l}\text { On soil in open places } \\
\text { and rotten wood }\end{array}$ & $\begin{array}{l}\text { Moflong, Khasi Hills, } \\
\text { Meghalaya, } \\
\text { Darjeeling, West } \\
\text { Bengal }\end{array}$ & $\begin{array}{l}\text { Berkeley (1852a) Horak } \\
\text { (1980) }\end{array}$ \\
\hline 38. & $\begin{array}{l}\text { Boletus squamatus Berk. } \\
=\text { Boletellus squamatus (Berk.) } \\
\text { Singer }\end{array}$ & In woods & $\begin{array}{l}\text { Myrung, Khasi Hills, } \\
\text { Meghalaya }\end{array}$ & Berkeley (1852a) \\
\hline 39. & $\begin{array}{l}\text { Boletus subaestivalis Sagar \& } \\
\text { T.N. Lakh. }\end{array}$ & $\begin{array}{l}\text { Growing among plant } \\
\text { debris }\end{array}$ & Himachal Pradesh & Lakhanpal (1996) \\
\hline 40. & $\begin{array}{l}\text { Boletus thiersii T.N. Lakh. \& } \\
\text { Sagar }\end{array}$ & On clear soil & Himachal Pradesh & Lakhanpal (1996) \\
\hline 41. & Boletus ustalis Berk. & On rotten tree trunk & $\begin{array}{l}\text { Darjeeling, West } \\
\text { Bengal }\end{array}$ & Berkeley (1851a) \\
\hline 42. & $\begin{array}{l}\text { Boletus variipes } \text { Peck } \\
=\text { Boletus variipes } \text { var. variipes } \\
\text { Peck }\end{array}$ & $\begin{array}{l}\text { Growing solitary or } \\
\text { gregariously in } \\
\text { angiosperms forest soil }\end{array}$ & $\begin{array}{l}\text { Shimla, Himachal } \\
\text { Pradesh }\end{array}$ & $\begin{array}{l}\text { Lakhanpal and Sagar } \\
\text { (1989) }\end{array}$ \\
\hline 43. & $\begin{array}{l}\text { Boletus vermiculosus var. } \\
\text { thindii T.N. Lakh. \& Sagar }\end{array}$ & $\begin{array}{l}\text { Associated with } \\
\text { Quercus sp. }\end{array}$ & Himachal Pradesh & Lakhanpal (1996) \\
\hline 44. & $\begin{array}{l}\text { Boletus verrucarius Berk. } \\
=\text { Boletellus verrucarius } \\
\text { (Berk.) Singer }\end{array}$ & On ground & Sikkim & Berkeley (1854a) \\
\hline 45. & Boletus spp. & $\begin{array}{l}\text { On ground and } \\
\text { and in moist deciduous } \\
\text { forest }\end{array}$ & $\begin{array}{l}\text { Namakkal, Tamil } \\
\text { Nadu; Shimoga, } \\
\text { Karnataka }\end{array}$ & $\begin{array}{l}\text { Raja et al., (2011) } \\
\text { Swapna et al., (2008) }\end{array}$ \\
\hline 46. & $\begin{array}{l}\text { Borofutus dhakanus Hosen \& } \\
\text { Zhu L. Yang }\end{array}$ & $\begin{array}{l}\text { Under sal and } \\
\text { deciduous forest }\end{array}$ & Koderma, Jharkhand & Parihar et al., (2014) \\
\hline 47. & $\begin{array}{l}\text { Chalciporus piperatus (Bull.) } \\
\text { Bataille } \equiv \text { Boletus piperatus } \\
\text { Bull., }\end{array}$ & $\begin{array}{l}\text { Ectomycorrhizal on sal, } \\
\text { growing solitary in sal } \\
\text { forest }\end{array}$ & $\begin{array}{l}\text { Balibhasa, West } \\
\text { Bengal }\end{array}$ & $\begin{array}{l}\text { Shajahan and Samajpati } \\
\text { (1995) }\end{array}$ \\
\hline 48. & $\begin{array}{l}\text { Chalciporus rubinellus (Peck) } \\
\text { Singer } \equiv \text { Boletus rubinellus } \\
\text { Peck }\end{array}$ & $\begin{array}{l}\text { growing gregariously } \\
\text { on ground in coniferous } \\
\text { forests and amongst } \\
\text { grasses under tree, }\end{array}$ & $\begin{array}{l}\text { Mandi, Himachal } \\
\text { Pradesh and Jodhpur, } \\
\text { Rajasthan }\end{array}$ & $\begin{array}{l}\text { Lakhanpal and Sagar } \\
\text { (1989); } \\
\text { Chaouhan et al., (2010) }\end{array}$ \\
\hline 49. & Hemileccinum subglabripes & On soil & Moflong, Khasi Hills, & Berkeley (1854a) \\
\hline
\end{tabular}




\begin{tabular}{|c|c|c|c|c|}
\hline & $\begin{array}{l}\text { (Peck) } \\
\text { Halling=Pulveroboletus } \\
\text { flavipes } \text { (Berk.) E. Horak } \\
\text { =Boletus flavipes } \text { Peck }\end{array}$ & & Meghalaya & \\
\hline 50. & $\begin{array}{l}\text { Hortiboletus indorubellus K. } \\
\text { Das, D. Chakr., Baghela, S.K. } \\
\text { Singh \& Dentinger }\end{array}$ & $\begin{array}{l}\text { On ground in forest, } \\
\text { under Betula alnoides }\end{array}$ & Sikkim & Das, et al., (2016) \\
\hline 51. & $\begin{array}{l}\text { Leccinum ustale (Berk.) E. } \\
\text { Horak } \equiv \text { Boletus ustalis Berk. }\end{array}$ & On soil & $\begin{array}{l}\text { Khasi Hills, } \\
\text { Meghalaya and } \\
\text { Sikkim }\end{array}$ & $\begin{array}{l}\text { Berkeley }(1851 \mathrm{a}, \mathrm{b}) \\
\text { Horak }(1980)\end{array}$ \\
\hline 52. & $\begin{array}{l}\text { Octaviania longiana } \mathrm{S} . \\
\text { Ahmad }\end{array}$ & $\begin{array}{l}\text { On ground amongst } \\
\text { grasses }\end{array}$ & Rohtak, Haryana & Ahmad (1950) \\
\hline 53. & $\begin{array}{l}\text { Phylloporus septocystidiatus } \\
\text { C.K. Pradeep \& K.B. Vrinda }\end{array}$ & $\begin{array}{l}\text { In tropical forest under } \\
\text { Hopea parviflora and } \\
\text { Xanthophyllum } \\
\text { arnottianum }\end{array}$ & $\begin{array}{l}\text { Palode, Trivandrum, } \\
\text { Kerala }\end{array}$ & In Pradeep et al., (2015) \\
\hline 54. & $\begin{array}{l}\text { Pulveroboletus fragicolor } \\
\text { (Berk.) Singer } \\
\text { =Phaeogyroporus fragicolor } \\
\text { (Berk.) E. Horak =Boletus } \\
\text { fragicolor Berk. }\end{array}$ & From mountain & $\begin{array}{l}\text { Nunklow, Khasi Hills, } \\
\text { Meghalaya }\end{array}$ & $\begin{array}{l}\text { Berkeley (1852a) Horak } \\
\text { (1980) }\end{array}$ \\
\hline 55. & $\begin{array}{l}\text { Pulveroboletus shoreae Singer } \\
\& \text { B. Singh }\end{array}$ & $\begin{array}{l}\text { Ectomycorrhizal on sal, } \\
\text { growing solitary in sal } \\
\text { forest, }\end{array}$ & $\begin{array}{l}\text { Dehradun, } \\
\text { Uttarakhand and } \\
\text { Gidhani West Bengal }\end{array}$ & $\begin{array}{l}\text { Singer and Singh, 1971; } \\
\text { Shajahan and Samajpati } \\
\text { (1995) }\end{array}$ \\
\hline 56. & $\begin{array}{l}\text { Retiboletus kauffmanii } \\
\text { (Lohwag) N.K. Zeng \& Zhu } \\
\text { L. Yang =Boletus kauffmanii } \\
\text { Lohwag }\end{array}$ & $\begin{array}{l}\text { Under Lithocarpus sp., } \\
\text { broadleaf forest }\end{array}$ & $\begin{array}{l}\text { East Distr., Maenam } \\
\text { Top, alt. } 2315 \mathrm{~m}, \\
\text { Sikkim }\end{array}$ & Chakraborty et al., (2017) \\
\hline 57. & $\begin{array}{l}\text { Retiboletus ornatipus (Peck) } \\
\text { Manfr. Binder \& Bresinsky }\end{array}$ & On ground & North West of Sikkim & Das (2013b) \\
\hline 58. & $\begin{array}{l}\text { Rhodactina himalayensis } \\
\text { Pegler \& T.W.K. Young }\end{array}$ & $\begin{array}{l}\text { on soil under leaf litter, } \\
\text { in association with } \\
\text { roots of sal }\end{array}$ & Uttar Pradesh & Pegler and Young (1989) \\
\hline 59. & $\begin{array}{l}\text { Strobilomyces echinocephalus } \\
\text { Gelardi \& Vizzini }\end{array}$ & $\begin{array}{l}\text { Growing in Quercus } \\
\text { semecarpifolia and wild } \\
\text { Punica granatum forest }\end{array}$ & $\begin{array}{l}\text { Jammu and Kashmir, } \\
\text { Poonch, Haveli, } \\
\text { Kanuyian }\end{array}$ & Kour et al., (2013) \\
\hline 60. & $\begin{array}{l}\text { Strobilomyces floccopus (Fr.) } \\
\text { Karsten }\end{array}$ & $\begin{array}{l}\text { On broad-leaved or } \\
\text { coniferous woods }\end{array}$ & $\begin{array}{l}\text { Thiruvananthapuram, } \\
\text { Kerala }\end{array}$ & Vrinda, Pradeep (2014) \\
\hline 61. & $\begin{array}{l}\text { Strobilomyces kalimpongensis } \\
\text { Bose }\end{array}$ & On dead wood & Kolkata, West Bengal & Bose (1946) \\
\hline 62. & Strobilomyces mollis Corner & $\begin{array}{l}\text { Growing on } \\
\text { humicolous soil under } \\
\text { Pinus roxburghii and } P \text {. } \\
\text { wallichiana. }\end{array}$ & $\begin{array}{l}\text { Jammu \& Kashmir, } \\
\text { Poonch, Haveli, } \\
\text { Krishna Ghati }\end{array}$ & $\begin{array}{l}\text { Kour et al., (2013); } \\
\text { Lakhanpal (1996) }\end{array}$ \\
\hline 63. & Strobilomyces montosus Berk. & On soil & $\begin{array}{l}\text { Khasi Hills, } \\
\text { Meghalaya }\end{array}$ & Berkeley $(1851 a, b)$ \\
\hline 64. & Strobilomyces nigricans Berk. & On soil & $\begin{array}{l}\text { Darjeeling, West } \\
\text { Bengal and Khasi } \\
\text { Hills, Meghalaya }\end{array}$ & (Berkeley, 1852) \\
\hline 65. & $\begin{array}{l}\text { Strobilomyces polypyramis } \\
\text { Hook. f. }\end{array}$ & On rotten wood and soil & $\begin{array}{l}\text { Darjeeling, West } \\
\text { Bengal and Sikkim }\end{array}$ & Horak (1980) \\
\hline 66. & $\begin{array}{l}\text { Strobilomyces strobilaceus } \\
\text { (Scop.) Berk. }\end{array}$ & $\begin{array}{l}\text { Grows in association } \\
\text { with coniferous trees }\end{array}$ & Nagaland & Ao et al., (2016) \\
\hline 67. & Strobilomyces velutipes & On ground & From Mussoorie, & Lloyd (1925) \\
\hline
\end{tabular}




\begin{tabular}{|c|c|c|c|c|}
\hline & $\begin{array}{l}\text { Cooke \& Massee } \\
=\text { Strobilomyces indicus Lloyd }\end{array}$ & & $\begin{array}{l}\text { Uttarakhand and } \\
\text { Saharanpur, Uttar } \\
\text { Pradesh }\end{array}$ & \\
\hline 68. & $\begin{array}{l}\text { Suillellus luridus (Schaeff.) } \\
\text { Murrill } \equiv \text { Boletus luridus } \\
\text { Schaeff. }\end{array}$ & Growing in leaf litter & Southern Rajasthan & $\begin{array}{l}\text { Doshi and Mohammad } \\
\text { (2015) }\end{array}$ \\
\hline 69. & $\begin{array}{l}\text { Suillus furfuraceus (Berk.) E. } \\
\text { Horak } \equiv \text { Boletus furfuraceus } \\
\text { Berk. }\end{array}$ & $\begin{array}{l}\text { On ground under } \\
\text { Andromeda (Pieris sp.) } \\
\text { and Betula }\end{array}$ & $\begin{array}{l}\text { Lachen, Sikkim and } \\
\text { Meghalaya }\end{array}$ & $\begin{array}{l}\text { Berkeley, 1852); (Horak, } \\
\text { 1980) }\end{array}$ \\
\hline 70. & $\begin{array}{l}\text { Suillus luteus (L.) Roussel } \\
\equiv \text { Boletus luteus } \mathrm{L} \text {. }\end{array}$ & On elephant dung & Assam & Sarma et al., (2010) \\
\hline 71. & $\begin{array}{l}\text { Suillus spraguei (Berk. \& } \\
\text { M.A. Curtis) Kuntze } \equiv \text { Boletus } \\
\text { spraguei Berk. \& M.A. Curtis }\end{array}$ & $\begin{array}{l}\text { On ground, semi } \\
\text { evergreen and moist } \\
\text { deciduous forest }\end{array}$ & $\begin{array}{l}\text { Amarkantak, Madhya } \\
\text { Pradesh }\end{array}$ & Dwivedi et al., (2012) \\
\hline 72. & $\begin{array}{l}\text { Tylopilus areolatus (Berk.) } \\
\text { Henn. } \equiv \text { Boletus areolatus } \\
\text { Berk. }\end{array}$ & $\begin{array}{l}\text { Growing in open } \\
\text { pasture }\end{array}$ & $\begin{array}{l}\text { Kala-Panee, Khasi } \\
\text { Hills, Meghalaya }\end{array}$ & $\begin{array}{l}\text { Berkeley (1852b); } \\
\text { Manjula (1983) }\end{array}$ \\
\hline 73. & $\begin{array}{l}\text { Tylopilus chromapes } \text { (Frost) } \\
\text { A.H. Sm. \& Thiers } \equiv \text { Boletus } \\
\text { chromapes } \text { Frost }\end{array}$ & Growing on grassland & West Bengal & De (2006b) \\
\hline 74. & $\begin{array}{l}\text { Tylopilus himalayanus D. } \\
\text { Chakr., K. Das \& Vizzini }\end{array}$ & $\begin{array}{l}\text { Under Pinus sp. in } \\
\text { mixed forest and under } \\
\text { Cedrus deodara in } \\
\text { coniferous forest }\end{array}$ & $\begin{array}{l}\text { East District, Upper } \\
\text { Chandmari, Sikkim } \\
\text { and Champawat and } \\
\text { Pauri, Uttarakhand }\end{array}$ & Chakraborty et al., (2018) \\
\hline 75. & $\begin{array}{l}\text { Tylopilus indecisus (Peck) } \\
\text { Murrill }\end{array}$ & $\begin{array}{l}\text { Growing on soil under } \\
\text { oak forest }\end{array}$ & Kumaon, Uttaralhand & Harsh and Bisht, (1982b) \\
\hline 76. & Tylopilus neofelleus Hongo & $\begin{array}{l}\text { Under Castanopsis sp. } \\
\text { in temperate broadleaf } \\
\text { forest }\end{array}$ & $\begin{array}{l}\text { East district, } \\
\text { Fambonglo WLS, } \\
\text { Sikkim }\end{array}$ & Chakraborty et al., (2018) \\
\hline 77. & $\begin{array}{l}\text { Tylopilus plumbeoviolaceus } \\
\text { (Snell \& E.A. Dick) Snell \& } \\
\text { E.A. Dick =Boletus } \\
\text { plumbeoviolaceus } \text { Snell \& } \\
\text { E.A. Dick }\end{array}$ & $\begin{array}{l}\text { On ground in a pasture } \\
\text { surrounded by Cedrus } \\
\text { deodara forest }\end{array}$ & $\begin{array}{l}\text { Kullu, Himachal } \\
\text { Pradesh }\end{array}$ & $\begin{array}{l}\text { Sharma and Lakhanpal } \\
\text { (1988) }\end{array}$ \\
\hline 78. & $\begin{array}{l}\text { Tylopilus pseudoballoui K. } \\
\text { Das, D. Chakr \& Vizzini }\end{array}$ & Under Quercus spp. & $\begin{array}{l}\text { South District, } \\
\text { Maenam WLS, Sikkim }\end{array}$ & Chakraborty et al., (2018) \\
\hline 79. & $\begin{array}{l}\text { Xerocomellus chrysenteron } \\
\text { (Bull.) Šutara =Xerocomus } \\
\text { chrysenteron (Bull.) Quél. }\end{array}$ & $\begin{array}{l}\text { On ground, semi } \\
\text { evergreen and moist } \\
\text { deciduous forest; sub- } \\
\text { tropical semi-evergreen } \\
\text { forests }\end{array}$ & $\begin{array}{l}\text { Amarkantak, Madhya } \\
\text { Pradesh } \\
\text { Nagaland }\end{array}$ & $\begin{array}{l}\text { (Dwivedi et al., 2012) } \\
\text { Ao et al., (2016) }\end{array}$ \\
\hline 80. & $\begin{array}{l}\text { Xerocomus bakshii Singer \& } \\
\text { B. Singh }\end{array}$ & $\begin{array}{l}\text { On soil connected with } \\
\text { roots of Pinus } \\
\text { roxburghii }\end{array}$ & $\begin{array}{l}\text { Dehradun, } \\
\text { Uttarakhand }\end{array}$ & Singer and Singh, (1971) \\
\hline 81. & $\begin{array}{l}\text { Xerocomus delphinus (Hook. } \\
\text { f.) Manjula }\end{array}$ & On open places of earth & $\begin{array}{l}\text { Darjeeling, West } \\
\text { Bengal }\end{array}$ & $\begin{array}{l}\text { Berkeley (1951b); } \\
\text { Manjula (1983) }\end{array}$ \\
\hline 82. & $\begin{array}{l}\text { Xerocomus doodhcha K. Das, } \\
\text { D. Chakr., Baghela, S.K. } \\
\text { Singh \& Dentinger }\end{array}$ & $\begin{array}{l}\text { On ground in broadleaf } \\
\text { forest, of Lithocarpus } \\
\text { pachyphyllus }\end{array}$ & Sikkim & Das, et al., (2016) \\
\hline 83. & Xerocomus indicus Singer & - & from India & $\begin{array}{l}\text { Butler and Bisby (1960); } \\
\text { Singer (1948) }\end{array}$ \\
\hline 84. & $\begin{array}{l}\text { Xerocomus longistipitatus K. } \\
\text { Das, A. Parihar, D. Chakr. \& } \\
\text { A. Baghela }\end{array}$ & $\begin{array}{l}\text { On soil under under } \\
\text { Lithocarpus sp., } \\
\text { broadleaf forest }\end{array}$ & $\begin{array}{l}\text { Rabangla, alt. 1985m, } \\
\text { Sikkim }\end{array}$ & Chakraborty et al., (2017) \\
\hline
\end{tabular}


Stipe central 4-6 x 1-2cm across, bulbous at base or almost parallel, pale greyish violet in apical part, whitish brown at base, reticulate in the upper half, base sub-radiating, flesh firm, white unchanging. Basidia 26-28 x 5$7 \mu \mathrm{m}$ clavate, 4 -spored, hyaline. Pleurocystidia scattered 36-42 x 6-9 $\mu \mathrm{m}$, narrowly fusoid ventricose, smooth, thin walled; cheilocystidia similar to pleurocystidia. Hyphae 7-10 $\mu$ m wide; subcutis composed of interwoven hyphae, stipe cuticle of loosely interwoven clavate to ventricose, thin-walled, clamp-connection absent. Basidiospores olive brown, 5-2.5 x 2-1.2 $\mu \mathrm{m}$, ellipsoid, subfusiform, smooth walled, hilum distinct.

\section{Collection examined}

Mycorrhizal on sal, Amarkantak-Achanakmar Biosphere Reserve, Madhya Pradesh and Chhattisgarh, 24/07/2012, Mycology Herbarium, Tropical Forest Research Institute, Jabalpur TF 2786.

Total 84 species of family boletaceae reported from India were compiled and presented in Table 1 including 37 species of Boletus excluding two unidentified species. The next common genus is Strobilomyces represented by 9 species followed by Tylopilus (7 species), Boletellus (6 species), Xerocomus (5 species each), and Suillus (3 species) Chalciporus and Pulveroboletus (2 species each). Austroboletus malacensis var autroboletus was reported from Quercus sp., Pinus wallichiana and Cedrus deodara forest, Jammu \& Kashmir (Kumar and Sharma, 2011b); Chalciporus piperatus, an ectomycorrhizal fungus from sal forest, West Bengal (Shajahan and Samajpati, 1995) while Chalciporus rubinellus was reported from coniferous forests of Mandi, Himachal Pradesh (Lakhanpal and Sagar, 1989) and Jodhpur, Rajasthan (Chaouhan et al., 2010). Octaviania asterosperma and Octaviania longiana were reported from Rohtak
(Cunningham, 1942; Ahmad, 1950). Pulveroboletus shoreae, an ectomycorrhizal bolete with sal, was reported from Dehradun, Uttarakhand and Gidhani, West Bengal (Singer and Singh, 1971; Shajahan and Samajpati, 1995) and Rhodactina himalayensis from Uttar Pradesh (Pegler and Young, 1989). Tylopilus areolatus, and Tylopilus chromapes were reported from Meghalaya (Berkeley, 1852b; Manjula, 1983) and West Bengal (De, 2006b) while Tylopilus indecisus and Tylopilus plumbeoviolaceus were reported from Uttaralhand (Harsh and Bisht, 1982b) and Himachal Praedesh (Sharma and Lakhanpal, 1988). Xerocomus bakshii, X. delphinus and X. indicus were also reported from India (Berkeley, 1951b; Butler and Bisby, 1960; Singer, 1948; Singer and Singh, 1971; Manjula, 1983).

There is no record of occurrence of bolataceae in Andhra Pradesh, Maharashtra and Karnataka but local communities in these states and also of Goa, Kerala and Tamil Nadu cultivate popular edible mushrooms including Boletaceae which are safe for human consumption (www.maria-online.com (Boletus). Along with other popular edible mushrooms the ectomycorrhizhal boletes also reported from Western Ghats (Maharashtra, Karnataka, Goa, Kerala and Tamil Nadu) (www.nzdl.org/gsdlmod?).

Fungi accommodated in family Boletaceae were reported from different places of India, a list of 82 species is presented in Table 1. Boletaceous mushroom fungi were distributed in Himachal Pradesh followed by Meghalaya, West Bengal, Uttarakhand, Madhya Pradesh, Chhattisgarh, Sikkim, Jammu and Kashmir, Kerala, Nagaland, Uttar Pradesh, Haryana and Rajasthan (Figure 31). Boletellus ananas reported from Kerala growing under Holigarna arnottiana (Vrinda and Pradeep, 2014) for the first time it is being reported from sal forest of CG. This species is earlier 
reported growing beside old log of Pinus and oaks in South Carolina (Murrill, 1909) and also as forming ect0-mycorrhizal associations with eucalypts in Australia (Gardner and Malajczuk 1988). Other places of distribution include Malaya, Singapore, Borneo, Kinabalu and Mesilau (Corner, 1972; Mayor et al., 2008; McNabb, 1967; Yeh et al., 1982; Zhishu, 1993). Although this mushroom is used as a food in Mexico (Boa, 2004) but another field guide listed it as inedible or not recommended for eating (Bessette et al., 2007).

Boletellus chrysenteroides, probably ectomycorrhizal and reported to be associated with oaks and eastern hemlock and often found growing near well decayed oak stumps, usually growing alone. This fungus is widely distributed in North Carolina, USA, Aylmer and Ontario in Canada (Snell, 1936, 1941) and also for the first is being reported from Madhya Pradesh and Chhattisgarh. However, Boletellus corneri was earlier reported as Boletus fallax from sal forest of Amarkantaka, Madhya Pradesh (Pyasi et al., 2012). This mushroom is reported to be distributed in Malaya (Singapore) (Corner, 1972). Boletellus dissiliens was reported growing on soil or ground near base of Myrtaceae and Fagaceae and distributed in Singapore and Australia (Corner, 1972). Boletellus pseudochrysenteroides is reported mycorrhizal with hardwoods of beech and oaks and distributed in USA (Illinois, Michigan and Arizona) (Smith and Thiers, 1971).

Genus Boletus is very common amongst mushroom of family boletaceae and out of 50 known species from world 37 species are reported from India (Table 1). Boletus edulis was reported growing on humicolous soil in coniferous forest of Jammu \& Kashmir (Kumar and Sharma 2011b). It was reported as forming ectomycorrhizal association with sal, Syzygium cuminii and growing on dead wood logs in Madhya Pradesh, Chhattisgarh, Jammu \& Kashmir, Jharkhand, Nagaland and Assam (Kumar and Sharma, 2011b; Srivastava et al., 2012; Ao et al., 2016; Sarma et al., 2010). The present article reports it for the first from sal forest of Madhya Pradesh and Chhattisgarh. Boletus lakhanpalii is recently reported from Sikkim (Das et al., 2015). Besides sal, other known tree species reported in literature under which this mushroom can grow and form ectomycorrhizal associations includes, Hopea ponga, H. parviflora, Vateria indica, and Diospyros malabarica. B. edulis is distributed worldwide and also reported from moist deciduous forests of India as well as in the forests of Arunachal Pradesh (Adhikary et al., 1999).

Austroboletus olivaceoglutinosus forming ecto-mycorrhizal association with Tsuga dumosa in forest of Sikkim (Das and Dentinger, 2015), it is the only species of the genus reported from India. Genus Chalciporus is represented by two species in India, $C$. piperatus which form ectomycorrhiza with sal in forest of West Bengal (Shajahan and Samajpati, 1995) and the another species, C. rubinellus is reported growing gregariously in coniferous forests and amongst grasses under tree from Mandi, Himachal Pradesh and Jodhpur, Rajasthan (Lakhanpal and Sagar, 1989; Chaouhan et al., 2010). Hemileccinum subglabripes is the only species under this genus recorded from Moflong, Khasi Hills, Meghalaya (Berkeley, 1854a). The species was earlier known as Pulveroboletus flavipes and Boletus flavipes. One species of Leccinum, L. ustale was reported from Khasi mountain, Meghalaya and from Sikkim (Berkeley (1851a, b; Horak, 1980) the species was earlier known as Boletus ustalis. Octaviania longiana was the only species of boletes reported from Rohtak, Haryana (Ahmad, 1950) while Phylloporus 
septocystidiatus is reported from tropical forest under Hopea parviflora and Xanthophyllum arnottianum from Palode, Thiruvanthapuram, Kerala (in Pradeep et al., 2015). Two species of Pulveroboletus, namely $P$. fragicolor and $P$. shoreae were reported India. $P$. fragicolor is reported from Nunklow, mountain Khasi, Meghalaya (Berkeley, 1852a; Horak, 1980). P. shoreae was reported to form ectomycorrhizal association with sal in forest at Dehradun, Uttarakhand (Singer and Singh, 1971) and the species is growing solitary in sal forest of Gidhani, West Bengal (Shajahan and Samajpati, 1995). Two species of Retiboletus, $R$. kauffmanii earlier known as Boletus kauffmanii was reported from broadleaf forest under Lithocarpus sp., from Maenam, Sikkim (Chakraborty et al., 2017) while second species was $R$. ornatipus which also occurred in Sikkim (Das, 2013b). One species, Rhodactina himalayensis was recorded growing in leaf litter and in association with sal trees in a forest of Uttar Pradesh (Pegler and Young, 1989).

Strobilomyces species have wide distribution from north to southern India and nine species have been reported including some recent reports. S. echinocephalus occur under Quercus semecarpifolia and wild Punica granatum forest and $S$. mollis grew on humicolous soil under Pinus roxburghii and $P$. wallichiana in Jammu and Kashmir (Kour et al., 2013; Lakhanpal, 1996). S. floccopus was reported from broad-leaved forests or coniferous woods from Thiruvananthapuram, Kerala (Vrinda and Pradeep, 2014). S. kalimpongensis occur on dead wood in Kolkata, West Bengal (Bose, 1946). S. montosus and S. nigricans were reported from Khasi Hills, Meghalaya and Darjeeling, West Bengal (Berkeley, 1851a, b; 1852). $S$. polypyramis was found on rotten wood and soil in Darjeeling, West Bengal and Sikkim (Horak, 1980). S. strobilaceus was recently reported growing in association with coniferous trees in Nagaland (Ao et al., 2016). S. velutipes was reported from Mussoorie, Uttarakhand and Saharanpur, Uttar Pradesh (Lloyd, 1925).

Suillellus luridus, earlier known as Boletus luridus was reported growing in leaf litter in forest of Southern Rajasthan (Doshi and Mohammad, 2015). Three species of Suillus were reported from Sikkim, Meghalaya, Assam and Madhya Pradesh including $S$. furfuraceus grew under Pieris and Betula, $S$. luteus on elephant dung and S. spraguei from semi evergreen and moist deciduous forest (Berkeley, 1852; Dwivedi et al., 2012; Horak, 1980; Sarma et al., 2010).

Seven species of genus Tylopilus were reported from northern and north eastern India these include: $T$. areolatus grew in open pasture at Khasi Hills in Meghalaya (Berkeley, 1852b; Manjula, 1983), $T$. chromapes from grassland of West Bengal (De, 2006b), T. himalayanus from Pinus sp. and Cedrus deodara forests of Sikkim and Uttarakhand; $T$. neofelleus grew under Castanopsis sp. and T. pseudoballoui under Quercus spp., Sikkim (Chakraborty et al., 2018). T. indecisus collected from soil surface under oak forest of Kumaon, Uttarakhand (Harsh and Bisht, 1982b) and $T$. plumbeoviolaceus from pasture surrounded by Cedrus deodara forest Himachal Pradesh was reported (Sharma and Lakhanpal, 1988).

Xerocomellus chrysenteron was recorded from semi evergreen and moist deciduous forest of Amarkantak, Madhya Pradesh and Nagaland (Dwivedi et al., 2012; Ao et al., 2016). X. bakshii was connected with roots of Pinus roxburghii in forest at Dehradun, Uttarakhand (Singer and Singh, 1971). $X$. delphinus was recorded from open places of earth from Darjeeling, West Bengal (Berkeley, 1951b; Manjula, 1983). Two 
species were recently described from Sikkim; $X$. doodhcha from broadleaf forest of Lithocarpus pachyphyllus and $X$. longistipitatus from the soil under Lithocarpus sp. (Das, et al., 2016; Chakraborty et al., 2017). Xerocomus indicus was also recorded from India (Butler and Bisby, 1960; Singer, 1948).

Total 84 species of Boletaceae were recorded from India. Boletus species are the most common followed by Strobilomyces, Tylopilus, Boletellus, Xerocomus, Suillus, Chalciporus, Retiboletus and Pulveroboletus. The most common bolete mushroom representing state is Himachal Pradesh in India followed by Sikkim, Meghalaya, West Bengal, Madhya Pradesh, Chhttisgarh and Kerala. Boletellus ananas, $B$. chrysenteroides, $B$. dissiliens, $B$. pseudochrysenteroides, $B$. corneri and Boletus edulis were recorded for the first time from Sal forest of Central India (Chhattisgarh and Madhya Pradesh).

\section{Acknowledgement}

The authors are thankful to Dr. G. Rajeshwar Rao, Director, Tropical Forest Research Institute, Jabalpur for providing the research facilities. The work presented was conducted under project ID No. 224/TFRI/2016/Patho1(22) funded by Indian Council of Forestry Research \& Education (ICFRE), Dehradun.

\section{References}

Adhikary RK, Baruah P, Kalita P, Bordoloi D (1999). Edible mushrooms growing in forests of Arunachal Pradesh. Advances in Horticulture and Forestry. 6: 11923.

Ahmad S (1950). Studies on Gasteromycetes V. Sydowia 4(1-6): 124-129.

Ao T, Seb J, Ajungla T, Deb CR (2016). Diversity of wild mushrooms in
Nagaland, India. Open Journal of Forestry 6: 404-419.

Berkeley MJ (1851a). Decades of fungi. Decades XXXII, XXXIII. Sikkim Himalaya fungi, collected by Dr. J.D. Hooker. Hooker's Journal of Botany and Kew Garden Miscellany 3: 39-49.

Berkeley MJ (1851b). Decades of fungi. Decades XXXIV. Sikkim Himalaya fungi, collected by Dr. J.D. Hooker. Hooker's Journal of Botany and Kew Garden Miscellany 3: 77-84.

Berkeley MJ (1852a). Decades of fungi. Decades XXXVII-XXXVIII. Sikkim and Khassya fungi. Hooker's Journal of Botany and Kew Garden Miscellany 4: 97-107.

Berkeley MJ (1852b). Decades of fungi. Decades XXXIXI-XL. Sikkim and Indian fungi. Hooker's Journal of Botany and Kew Garden Miscellany 4: 130-142.

Berkeley MJ (1854a). Decades of fungi. Decades XLI- XLIII. Indian fungi. Hooker's Journal of Botany and Kew Garden Miscellany 6: 129-143.

Bessette AE, Roody WC, Bessette AR (2007). Mushrooms of the Southeastern United States. Syracuse, NY: Syracuse University Press. p. 208.

Bhavanidevi S, Nair MC (1983). Addition to Indian Agaricales. In: Indian Mushroom Science-II (eds TN Kaul, BM Kapoor). CSIR, New Delhi, 271- 274.

Boa E. (2004). Wild edible fungi: A global overview of their use and importance to people (Non-Wood Forest Products). Food \& Agriculture Organization of the UN. pp. 118-19.

Bose SR (1946). Polyporaceae of Bengal XI. Journal of the Departmental Science Calcutta University. 2: 53-87

Butler EJ, Bisby GR (1960). The Fungi of India (revised by R.S. Vasudeva). Page(s): 1-552 
Chakraborty D, Das K, Parihar A, Adhikari S, Mukherjee SK, Karmakar J, Bandyopadhyay TK (2017) Morphology and phylogeny of Retiboletus kauffmanii (boletaceae): a new record of wild mushroom from Indian Himalaya. Indian Journal of Plant Sciences 6(1):5-11

Chakraborty D, Parihar A, Nikita Mehta, Baghela A and Das K (2017). A new species Xerocomus (Boletaceae) from India. Mycosphere 8(1): 44-50.

Chakraborty D, Vizzini A, Das K (2018).Two new species and one new record of the genus Tylopilus (Boletaceae) from Indian Himalaya with morphological details and phylogenetic estimations, MycoKeys 33: 103-124.

Chaouhan R, Kaur S, Gehlot P (2010). Some new records of Mushroom from India. Journal of Mycology and Plant Pathology 40(4): 550-554.

Corner EJH (1972). Boletus in Malaysia. Singapore: Botanic Gardens

Corner EJH, Lim Bian Han (1972). Boletus fallax sp. nov. Boletus in Malaysia, Government Printer, 263 Pp.

Cunningham GH (1942). The Gasteromycetes of Australia and New Zealand. Printed privately by John McIndoe, 76 Vogel Street, Dunedin, New Zealand.

Dar GH, Ganai NA, Beig MA, Ahangar FA, Sofi TA (2010). Biodiversity of macrofungal conifer dominated forests of Kashmir, India. Journal of Mycology and Plant Pathology 40(2): 169-171.

Das K (2013a). Boletus rubripes Thiers, a new record of wild mushroom from Sikkim (India). Taiwania 58(2): 136139.

Das K (2013b). New distribution record of Retiboletus ornatipus (Peck) Manfr. Binder \& Bresinsky (Boletaceae) from North West of Sikkim. Indian Journal of Plant Science 2(1): 1-5. 11-19.
Das K, Chakraborty D, Baghela A, Singh SK, Dentinger BTM (2015). Boletus lakhanpalii, a new species in Boletaceae from Sikkim (India) with uncertain phylogenetic placement. Sydowia 67: 11-19.

Das K, Chakraborty D, Baghela A, Singh SK, Dentinger BTM (2016). New species of xerocomoid boletes (Boletaceae) from Himalayan India based on morphological and molecular evidence. Mycologia. 108(4):753-764

Das K, Chakraborty D, Vizzini A (2017) Morphological and phylogenetic evidences unveil a novel species of Gyroporus (Gyroporaceae, Boletales) from Indian Himalaya. Nordic Journal of Botany 35: 669-675.

Das K, Dentinger BT (2015). Austroboletus olivaceoglutinosus, a new mushroom species from Sikkim, India with a distinctive green, glutinous pileus. Kew Bulletin 70: 15: 1-7.

De AB (2006). Two new additions to Indian Boletaceae. Journal of Natural History 2(1): 11-16.

Doshi A and Mohammad I (2015). Litter decomposing fungi in the forest of southern Rajasthan, India. Department of Plant Pathology, Rajasthan College of Agriculture, MPUAT, Udaipur, Rajasthan 1p.

Dwivedi S, Tiwari MK, Chauhan UK and Pandey AK (2012). Biodiversity of mushrooms of Amarkantak Biosphere Reserve Forest of Central India. International Journal of Pharmaceutical and Life Sciences 3(1): 1363-1367.

Florence EJM (2004). Biodiversity Documentation for Kerala, Part 2: Microorganisms (Fungi). KFRI Handbook No.17. Kerala Forest Research Institute, Peechi, Kerala, India. 
Gardner JH, Malajczuk N (1988). Recolonisation of rehabilitated bauxite mine sites in Western Australia by mycorrhizal fungi. Forest Ecology and Management 24: 27-42

Harsh NSK, Bisht NS (1982b). Boletaceae of Kumaun Hills, India. Current Science 52: 316-317.

Harsh NSK, Bisht NS (1985). Boletaceae of Kumaun Hills, India-II. Mycologia Helvetica 1: 443-445.

Kamat NM, Phadte KR, Kalangutkat JR, D 'silva NV (2009). Ecological, phonological, biochemical significance of biomodal fruiting of Boletus alutaceus Morgan an ectomycorrhizal partner of Ficus benghalensis L. Nature Proceedings doi:10.1038/npre.2009.2835.1

Kirk PM, Cannon PF, Minter DW, Stalpers JA (2008). Ainsworth and Bisby's Dictionary of the Fungi 10th Edition. 9th Edition ed. Wallingford, UK: CABI Publishing

Kour H, Kumar S, Sharma YP 2013 - Two species of Strobilomyces from Jammu and Kashmir, India. Mycosphere 4(5), 1006-1013

Kumar S, Sharma YP (2011b). Additions to Boletales from Jammu and Kashmir. Journal of Mycology and Plant Pathology 41(4): 579-583.

Lakhanpal TN (1996) Mushrooms of India: Boletaceae, vol. I. APH Publishing Corporation, New Delhi, 170 pp.

Lakhanpal TN, Sagar A (1989). Fleshy fungi of N.W. Himalayas - XIV. Three species of Boletus new to India. Kavaka 17(1-2): 32-37.

Lloyd CG (1925). Mycological Notes 74. Mycological Writings 7(74):1333-1348.

Manjula B (1983). A revised list of the agaricoid and boletoid Basidiomycetes from India and Nepal. Proceedings of the Indian Academy of Sciences (Plant Sciences) 92(2): 81-213.
Mayor JR, Fulgenzi TD, Henkel TW, Halling RE (2008). Boletellus piakaii sp nov and a new distribution record for Boletellus ananas var. ananas from Guyana. Mycotaxon. 105: 387-98

McNabb RFR (1967). "The Strobilomycetaceae of New Zealand". New Zealand Journal of Botany. 5 (4): 532-47

Mohanan C (2011). Macrofungi of Kerala. Kerala Forest Research Institute, Hand Book \# 27, Kerala, India, 597pp.

Murrill WA (1909). The Boletaceae of North America - 1. Mycologia 1(1): 4-18

Nelson SF. (2010). Bluing components and other pigments of Boletes. Fungi. 3(4): 11-14

Parihar A, Hembrom ME, Das K (2014). Borofutus dhakanus (Boletaceae) - an addition to Indian Mycobiota. NELUMBO 56: 342-345.

Pegler DN, Young TWK (1981) A natural arrangement of the Boletales, with reference to spore morphology. Transactions of the British Mycological Society 76(1): 103-146

Pradeep CK, Vrinda KB, Shibu P, Varghese, Arun Kumar TK (2015). A new species of Phylloporus (Agaricales, Boletaceae) from India. Phytotaxa 222: 269-274.

Pyasi A, Soni KK, Verma RK (2012). A new record of Boletus fallx from India. Journal of Mycology and Plant Pathology 42(1): 172-173.

Raja A, Nandakumar K, Gowrisankar K, Sivashanmungam $\mathrm{M}$, Jayabalan $\mathrm{N}$ (2011). A check list of terrestrial fungi in and around Cauvery River bed of Velur Namakkal District Tamil Nadu, India. Journal of Basic and Applied Biology 5(1\&2): 209-213.

Sarma TC, Sarma I, Patiri BN (2010). Wild edible mushrooms used by some ethnic tribes of Western Assam. The Bioscan 3: 613-625. 
Shajahan M, Samajpati N (1995). Ectomycorrhizal fungi of Shorea robusta Gf from West Bengal. Journal of Mycopathological Research 33(2): 105-117.

Sharma AD, Jandaik CL, Munjal RL, Seth PK (1978). Some fleshy fungi from Himachal Pradesh -I. Indian Journal of Mushroom 4: 1-4.

Sharma R, Lakhanpal TN (1988). Fleshy fungi of N.W. Himalayas - XVII. The genus Tylopilus (Boletaceae). Kavaka 16(1-2): 36-39.

Singer R (1948). New and interesting species of Basidiomycetes. II. Papers of the Michigan Academy of Sciences 32: 103 150.

Singer R, Singh B (1971). Two new ectotroph-forming boletes from India. Mycopathologia et Mycologia applicata 43: 25-33.

Smith AH, Thiers HD (1971). The Boletes of Michigan. Pp.1-428

Snell WH (1936). Notes on boletes - V. Mycologia 28(5): 463-75.

Snell WH (1941). The genera of the Boletaceae. Mycologia 33 (4): 415-23

Srivastava AK, Prabhat Fr, Kennedy Soreng SJ (2012). An effort to domesticate wild edible mushrooms growing in the forest of Jharkhand. International Journal of Recent Trends in Science and Technology 3(3): 88-92.

Swapna S, Syed Abrar and Krishnappa M. (2008). Diversity of macrofungi in semi-evergreen and moist deciduous forest of Shimoga district-Karnataka, India. J Mycol Pl Pathol 38(1): 21-26.

Tiwari CK, Parihar J, Verma RK, Prakasham U (2013) Atlas of wood decaying fungi of central India. Tropical Forest Research Institute, Jabalpur, 166p.

Verma RK, Sharma Nidhi, Soni KK, Jamaluddin (2008) Forest Fungi of Central India. International Book Distributing Co. Lucknow, 418p.

Vrinda KB, Pradeep CK (2014). Wild edible mushrooms from Kerala forests-a source of food \& income. Final project report submitted to department of planning \& economic affairs, WGDP, Govt. Of Kerala, Thiruvanthapuram, Kerala, 124p + IX colored plates.

Wu G, Feng B, Xu J, Zhu X-T, Li Y-C, Zeng N-K, Hosen MI, Yang ZL (2014). Molecular phylogenetic analyses redefine seven major clades and reveal 22 new generic clades in the fungal family Boletaceae. Fungal Diversity. 69 (1): 93-115.

Wu G, Zhao K, Li Y-C, Zeng N-K, Feng B, Halling R, Yang ZL (2015). Four new genera of the fungal family Boletaceae. Fungal Diversity. 81: 1-24.

www.maria-online.com (Boletus) page visited on $03 / 05 / 2018$

www.nzdl.org/gsdlmod? Biodiversity in the Western Ghats: An information kit. 6.7 Edible mushrooms (page visited 0n 04/05/2018)

Yeh K-W, Chen Z-C (1982). "The Boletes of Taiwan 3". Taiwania. 27: 52-63.

Zang, Mu, Tai-Hui Li \& Ronald H. Petersen (2001). Five new species of Boletaceae from China. Mycotaxon 80: 481-487.

Zhishu B, Zheng G, Taihui L (1993). The Macrofungus Flora of China's Guangdong Province (Chinese University Press). New York: Columbia University Press. pp. 461-62.

\section{How to cite this article:}

Verma R. K. and Vimal Pandro. 2018. Distribution of Boleteceous Mushrooms in India, Some New Records from Sal Forest of Central India. Int.J.Curr.Microbiol.App.Sci. 7(06): 1694-1713. doi: https://doi.org/10.20546/ijcmas.2018.706.201 